\title{
A structural result for hypergraphs with many restricted edge colorings
}

\author{
Hanno Lefmann, Yury Person* and Mathias Schacht \\ In honor of Joel Spencer's 60+ birthday
}

For $k$-uniform hypergraphs $F$ and $H$ and an integer $r \geq 2$, let $c_{r, F}(H)$ denote the number of $r$-colorings of the set of hyperedges of $H$ with no monochromatic copy of $F$ and let $c_{r, F}(n)=$ $\max _{H \in \mathcal{H}_{n}} c_{r, F}(H)$, where the maximum runs over the family $\mathcal{H}_{n}$ of all $k$-uniform hypergraphs on $n$ vertices. Moreover, let $\operatorname{ex}(n, F)$ be the usual Turán function, i.e., the maximum number of hyperedges of an $n$-vertex $k$-uniform hypergraph which contains no copy of $F$.

In this paper, we consider the question for determining $c_{r, F}(n)$ for arbitrary fixed hypergraphs $F$ and show

$$
c_{r, F}(n)=r^{\operatorname{ex}(n, F)+o\left(n^{k}\right)}
$$

for $r=2,3$. Moreover, we obtain a structural result for $r=2,3$ and any $H$ with $c_{r, F}(H) \geq r^{\operatorname{ex}(|V(H)|, F)}$ under the assumption that a stability result for the $k$-uniform hypergraph $F$ exists and $|V(H)|$ is sufficiently large. We also obtain exact results for $c_{r, F}(n)$ when $F$ is a 3 - or 4-uniform generalized triangle and $r=2,3$, while $c_{r, F}(n) \gg r^{\operatorname{ex}(n, F)}$ for $r \geq 4$ and $n$ sufficiently large.

\section{Introduction and results}

\subsection{Introduction}

For real constants $\alpha, \beta$, and a non-negative constant $\xi$ we sometimes write $\alpha=\beta \pm \xi$ if $\beta-\xi \leq \alpha \leq \beta+\xi$. For a positive integer $\ell$, we denote by $[\ell]$ the set $\{1, \ldots, \ell\}$. For a set $V$ and an integer $k \geq 1$, let $[V]^{k}$ be the set of all $k$ element subsets of $V$. We may drop one pair of brackets and write $[\ell]^{k}$ instead of $[[\ell]]^{k}$. A subset $H^{(k)} \subseteq[V]^{k}$ is a $k$-uniform hypergraph on the vertex set $V$.

*Supported by GIF grant no. I-889-182.6/2005. Part of this work was done while visiting the Institute for Pure and Applied Mathematics at UCLA, partly supported through NSF. 
We identify hypergraphs with their sets of hyperedges. For a given $k$-uniform hypergraph $H^{(k)}$, we denote by $V\left(H^{(k)}\right)$ and $E\left(H^{(k)}\right)$ its vertex set and its set of hyperedges, respectively. For $U \subseteq V\left(H^{(k)}\right)$, we denote by $H^{(k)}[U]$ the subhypergraph of $H^{(k)}$ induced on $U$ (i.e., $H^{(k)}[U]=H^{(k)} \cap[U]^{k}$ ). We set $e\left(H^{(k)}\right):=\left|E\left(H^{(k)}\right)\right|$ to be the number of hyperedges of $H^{(k)}$. We also write $H$ instead of $H^{(k)}$ to simplify the notation.

For $k$-uniform hypergraphs $F$ and $H$ and an integer $r$ let $c_{r, F}(H)$ denote the number of $r$-colorings of the set of hyperedges of $H$ with no monochromatic copy of $F$ and let

$$
c_{r, F}(n)=\max _{H \in \mathcal{H}_{n}} c_{r, F}(H)
$$

where the maximum runs over $\mathcal{H}_{n}$, the set of all $k$-uniform hypergraphs on $n$ vertices. We call $H$ an $F$-free hypergraph if $H$ contains no subhypergraph isomorphic to $F$. Moreover, let $\operatorname{ex}(n, F)$ be the Turán function, i.e., the maximum number of hyperedges of an $F$-free $n$-vertex $k$-uniform hypergraph. We say also that an $F$-free hypergraph $H$ on $n$ vertices is extremal for $F$ if $e(H)=\operatorname{ex}(n, F)$. Further set

$$
\pi_{F}:=\lim _{n \rightarrow \infty} \operatorname{ex}(n, F) /\left(\begin{array}{l}
n \\
k
\end{array}\right),
$$

and call $\pi_{F}$ Turán density of $F$.

Clearly, every coloring of the set of hyperedges of any extremal hypergraph $H$ for $F$ contains no monochromatic copy of $F$ and, consequently,

$$
c_{r, F}(n) \geq r^{\operatorname{ex}(n, F)}
$$

for all $r \geq 2$. On the other hand, if $\operatorname{Forb}_{F}(n)$ denotes the family of all labeled hypergraphs on the vertex set $[n]$ which contain no copy of $F$ as a subhypergraph, since every 2-coloring of the set of hyperedges of a hypergraph $H$, which contains no monochromatic copy of $F$, gives rise to a member of Forb $_{F}(n)$, e.g., consider always the subhypergraph in one of the two colors, we have

$$
c_{2, F}(n) \leq\left|\operatorname{Forb}_{F}(n)\right| .
$$

The family $\operatorname{Forb}_{F}(n)$ was studied by several researchers (see [8, 17, 18, $7,3,2,22,23,24,4])$. For example, it was shown in [23] that $\left|\operatorname{Forb}_{F}(n)\right| \leq$ $2^{\operatorname{ex}(n, F)+o\left(n^{k}\right)}$ for every fixed $k$-uniform hypergraph $F$, consequently,

$$
2^{\operatorname{ex}(n, F)} \leq c_{2, F}(n) \leq 2^{\operatorname{ex}(n, F)+o\left(n^{k}\right)}
$$


In the graph case, when $F=K_{\ell}$ is a graph clique, Yuster [33] (for $\ell=3$ ) and Alon, Balogh, Keevash and Sudakov [1] (for arbitrary fixed $\ell)$ closed the gap in (1) and showed, that the lower bound is the correct order of $c_{2, K_{\ell}}(n)$, i.e., $c_{2, K_{\ell}}(n)=2^{\operatorname{ex}\left(n, K_{\ell}\right)}$ for $n$ sufficiently large, which was conjectured by Erdős and Rothschild (see [6]). Moreover, Alon et al. showed that $c_{3, K_{\ell}}(n)=3^{\operatorname{ex}\left(n, K_{\ell}\right)}$ for $n$ sufficiently large and in both cases $r=2,3$ we have

$$
c_{r, K_{\ell}}(H)=c_{r, K_{\ell}}(n)=r^{\mathrm{ex}\left(n, K_{\ell}\right)}
$$

only when $H$ is the $(\ell-1)$-partite Turán graph, $\ell \geq 3$. In fact, it was shown in [1] that the same result holds for $\ell$-chromatic graphs which contain a colorcritical edge. Furthermore, it was observed in [1] that $c_{r, K_{\ell}}(n) \gg r^{\operatorname{ex}\left(n, K_{\ell}\right)}$ for $r \geq 4$. Recently, Pikhurko and Yilma [27] determined the graphs that yield $c_{4, K_{3}}(n)$ and $c_{4, K_{4}}(n)$.

Rödl and the authors [21] showed that a similar result holds, when $F$ is the 3-uniform hypergraph of the Fano plane, i.e., the unique 3-uniform hypergraph with 7 hyperedges on 7 vertices in which every pair of vertices is contained in precisely one hyperedge. More precisely, it was shown that for large $n$ and $r=2,3$ one has for every 3-uniform hypergraph $H$ on $n$ vertices that $c_{r, F}(H) \leq r^{\operatorname{ex}(n, F)}$. Moreover, the only 3-uniform hypergraph $H$ on $n$ vertices with $c_{r, F}(H)=r^{\operatorname{ex}(n, F)}$ is the extremal hypergraph for $F$, i.e., $H$ is isomorphic to $B_{n}$, the balanced, complete, bipartite hypergraph on $n$ vertices (see $[14,12])$.

\subsection{Main results}

We continue to study $c_{r, F}(n)$ for hypergraphs $F$. We obtain a general result related to (1). Roughly speaking, we show that for a natural class of hypergraphs $F$, any hypergraph $H$ for which many $F$-free colorings exist must disclose a special structure. To state this result precisely, we use the notions of $\varepsilon$-closeness and $s$-stability.

We say that two $k$-uniform hypergraphs $H_{1}$ and $H_{2}$ on $n$ vertices are $\varepsilon$-close if there exists a bijective function $\varphi: V\left(H_{1}\right) \longrightarrow V\left(H_{2}\right)$ such that $\left|E\left(H_{1}\right) \Delta \varphi\left(E\left(H_{2}\right)\right)\right| \leq \varepsilon n^{k}$, where $\Delta$ denotes the symmetric difference.

Recall the notion of $s$-stability as introduced by Pikhurko [25].

Definition 1 (s-stability). Let $F$ be a $k$-uniform hypergraph. Call $F s$ stable, if for every $\varepsilon>0$ there exists an $\omega>0$ and an integer $n_{0}$ such that for arbitrary $F$-free $k$-uniform hypergraphs $H_{1}, \ldots, H_{s+1}$ each of the same order $n \geq n_{0}$ and each having at least $\pi_{F}\left(\begin{array}{l}n \\ k\end{array}\right)-\omega n^{k}$ hyperedges, there are two which are $\varepsilon$-close. 
In the graph case, every $F$-free graph on $n$ vertices with roughly $\pi_{F}\left(\begin{array}{l}n \\ 2\end{array}\right)$ many edges is $\varepsilon$-close to its extremal graph, which is a result of Erdös and Simonovits [31]. In particular, 1-stability summarizes this scenario. However, for hypergraphs such a result was not obtained and it is believed that in general it even fails for $k \geq 3$ and $k$-uniform hypergraphs. We consider those hypergraphs $F$ for which such a stability result exists.

The structural result which we prove in this paper is the following.

Theorem 2. Let $k, s \in \mathbb{N}, k \geq 2$ and $r=2$ or 3 . Let $F$ be a $k$-uniform hypergraph, such that $\pi_{F}>0$.

Then, for every $\varepsilon>0$ there exists an $n_{0} \in \mathbb{N}$ such that for every $n \geq n_{0}$ it is

$$
c_{r, F}(n) \leq r^{\operatorname{ex}(n, F)+\varepsilon n^{k}}
$$

Furthermore, suppose that $F$ is s-stable. Then, among any $s+1 k$ uniform hypergraphs $H_{1}, \ldots, H_{s+1}$ on $n \geq n_{0}$ vertices that satisfy $c_{r, F}\left(H_{i}\right) \geq$ $r^{\operatorname{ex}(n, F)}$ for every $i \in[s+1]$, there exist two which are $\varepsilon$-close.

Note that for the general upper bound (2) on the number $c_{r, F}(n)$, the property $s$-stability is not required. The upper bound (2) also holds for those hypergraphs $F$ with $\pi_{F}=0$ and an arbitrary fixed number of colors. This is a triviality due to $\pi_{F}=0$.

For certain hypergraphs we also obtain a precise result in the spirit of $[1,33,21]$. For any positive integers $n \geq \ell \geq k$, we define the Turán hypergraph $\mathcal{T}_{\ell}^{(k)}(n)$ as follows. Partition the vertex set $[n]$ into $\ell$ mutually disjoint subsets $V_{1}, \ldots, V_{\ell}$ of sizes as equal as possible, i.e., they differ in size by at most 1 . Then, consider as hyperedges all $k$-element subsets of $[n]$ that intersect every partition class $V_{i}, i \in[\ell]$, in at most one vertex. It is easy to check that the Turán hypergraph $\mathcal{T}_{\ell}^{(k)}(n)$ contains the maximum possible number of hyperedges with the property that every hyperedge intersects every class $V_{i}, i \in[\ell]$, in at most one vertex, and is unique up to isomorphism.

For an integer $k \geq 2$ define the generalized triangle $T_{k}$ as follows. This $k$-uniform hypergraph $T_{k}=(V, E)$ has the vertex set $V=[2 k-1]$ and its set $E$ of three hyperedges is given by

$$
E=\{\{1, \ldots, k\},\{1, \ldots, k-1, k+1\},\{k, k+1, \ldots, 2 k-1\}\} .
$$

Thus, the first two hyperedges have $(k-1)$ common vertices, while the third hyperedge contains the symmetric difference of the first two, and intersects each of these in precisely one vertex. Clearly, if $k=2$, then $T_{2}$ is a graph 
triangle $K_{3}$. For $k=3$ it was shown by Frankl and Füredi [9] and for $k=4$ by Pikhurko [26], that the Turán hypergraph $\mathcal{T}_{k}^{(k)}(n)$ is the unique extremal $T_{k}$-free hypergraph for $n$ sufficiently large. Moreover, Keevash and Mubayi [13] (case $k=3$ ) and Pikhurko [26] (case $k=4$ ) showed that $T_{k}$ is 1-stable. Our second result shows that for these cases we obtain precise results for $c_{r, T_{k}}(n)$ for $r=2,3$ and $k=3,4$.

Theorem 3. Let $k=3$ or 4 and $r=2$ or 3 . There exists an integer $n_{r, k}$, such that

$$
c_{r, T_{k}}(H) \leq r^{\operatorname{ex}\left(n, T_{k}\right)}
$$

for any $k$-uniform hypergraph $H$ on $n \geq n_{r, k}$ vertices. Moreover, if

$$
c_{r, T_{k}}(H)=r^{\operatorname{ex}\left(n, T_{k}\right)}
$$

then $H$ is isomorphic to the Turán hypergraph $\mathcal{T}_{k}^{(k)}(n)$.

For $k=5,6$, the hypergraph $\mathcal{T}_{k}^{(k)}(n)$ is not extremal for $T_{k}$ anymore [10]. Furthermore one can also extend the constructions from [10] to show that for $k \geq 7, \mathcal{T}_{k}^{(k)}(n)$ is not extremal for $T_{k}$ as well.

\subsection{Overview of the proof}

Our motivation is a better understanding of the approach of Alon, Balogh, Keevash and Sudakov [1]. Their proof strategy can be described as follows. One splits the proof into two parts. In the first part they obtain the following structural result. If, say, a graph $G$ on $n$ vertices admits at least $r^{\operatorname{ex}\left(n, K_{\ell}\right)}$ many edge colorings without a monochromatic complete graph $K_{\ell}$, then $G$ looks almost like the Turán graph $\mathcal{T}_{\ell-1}^{(2)}(n)$. In the second part of the argument they use backward induction. Assuming that $G$ is not the Turán graph, one consecutively removes vertices to derive an impossible fact about some subgraph $G^{\prime} \subseteq G$. Namely, that $c_{r, K_{\ell}}\left(G^{\prime}\right)>r\left(\begin{array}{c}\left|V\left(G^{\prime}\right)\right| \\ 2\end{array}\right)$, which is clearly a contradiction, as any graph on $\left|V\left(G^{\prime}\right)\right|$ vertices may have at most $\left(\begin{array}{c}\left|V\left(G^{\prime}\right)\right| \\ 2\end{array}\right)$ edges.

We generalize the first part of the argument for stable hypergraphs $F$. Before we sketch our approach, we discuss the idea of Alon et al. [1]. To prove such a structural result one fixes some edge-coloring of $G$ without a monochromatic copy of $F$, and one applies the colored version of the regularity lemma to $G$ (see for example [19]). Then, almost all edges are contained in $\varepsilon$-regular pairs of sufficiently large density. This allows to concentrate on 
the cluster graphs defined for every color. The idea now is to consider the new cluster graph that consists of the $\varepsilon$-regular pairs in all colors. Furthermore, if one could apply to that cluster graph a stability-type result of Erdös and Simonovits [31], then this would give also a partition of the underlying graph, as then the number of the other $\varepsilon$-regular pairs that are contained inside the partition classes would be small. However, being unable to apply the stability result for any coloring, as the cluster graph contains too few edges, one can then derive a contradiction by bounding $c_{r, K_{\ell}}(G)$ from above by $r^{\operatorname{ex}\left(|V(G)|, K_{\ell}\right)-1}$.

To get an approximate result for 3-uniform hypergraphs with $F$ being the Fano plane, we used the so-called weak hypergraph regularity lemma (see e.g. [5, 11, 32]), which is a straightforward generalization of Szemerédi's regularity lemma for graphs. This version has recently been proved [15] to be compatible (i.e., admits a counting lemma) with linear hypergraphs, i.e., those hypergraphs whose hyperedges intersect pairwise in at most one vertex. In this setting the notion of the cluster hypergraph is the same as for graphs.

For the general hypergraph case, we use the regularity lemma of Rödl and Schacht from [29] together with some form of the corresponding counting lemma proved also by these authors in [28]. However, applications of this lemma yield partitions with a more complicated structure. In particular, there is no such simple notion of the cluster hypergraph as in the graph case. Nevertheless, one way to obtain a similar structural result is still to apply some appropriate stability result, but this time not to the cluster hypergraph, but to an underlying hypergraph. Indeed, in the approach of Alon et al. [1] instead of applying the stability result to the cluster graph one could do the following: once one can apply the stability result to the cluster graph, whose edges correspond to $\varepsilon$-regular pairs of sufficiently large density in every color, one may ignore the colors, and replace each such $\varepsilon$-regular pair by the complete bipartite graph, which is clearly $\varepsilon$-regular. Then we can apply the stability result, unless some copy of $K_{\ell}$ suddenly appears. This is impossible due to the fact that then the edges of this copy must correspond to $\varepsilon$-regular pairs, which are $\varepsilon$-regular in every color. Thus, these pairs form a copy of $K_{\ell}$ in the cluster graph which is a contradiction.

\subsection{Organization}

In the next section we provide the reader with notions related to the strong regularity lemma (colored version) of Rödl and Schacht. We mainly follow the notation from $[29,28]$ and include it here for completeness. Section 3 
is devoted to the proof of Theorem 2. Section 4 contains further notations used in the proof of Theorem 3, to which Section 5 is devoted. In Section 6 we present a construction showing that $c_{r, T_{k}}(n) \gg r^{\operatorname{ex}\left(n, T_{k}\right)}$ for $r \geq 4$ and $k=3,4$. We close with a few concluding remarks in Section 7 .

\section{Regularity lemma for hypergraphs}

Before we state the regularity and the counting lemmas [29, 28], we introduce some notation.

\subsection{Complexes}

A $k$-uniform clique of order $j$, denoted by $K_{j}^{(k)}$, is a complete $k$-uniform hypergraph on $j \geq k$ vertices consisting of all $\left(\begin{array}{l}j \\ k\end{array}\right)$ different $k$-subsets. Here, by $k$-subsets we mean $k$-element subsets.

Given integers $j \leq \ell$ and mutually disjoint vertex sets $V_{1}, \ldots, V_{\ell}$, we denote by $K_{\ell}^{(j)}\left(V_{1}, \ldots, V_{\ell}\right)$ the complete $\ell$-partite, $j$-uniform hypergraph (i.e., the set of all $j$-subsets $J \subseteq \bigcup_{i \in[\ell]} V_{i}$ satisfying $\left|V_{i} \cap J\right| \leq 1$ for every $i \in[\ell]$ ). If $\left|V_{i}\right|=m$ for every $i \in[\ell]$, then an $(m, \ell, j)$-hypergraph $H^{(j)}$ on $V_{1} \cup \cdots \cup V_{\ell}$ is any subset of $K_{\ell}^{(j)}\left(V_{1}, \ldots, V_{\ell}\right)$. Note that the vertex partition $V_{1} \cup \cdots \cup V_{\ell}$ is an $(m, \ell, 1)$-hypergraph $H^{(1)}$. For $j \leq i \leq \ell$ and a set $\Lambda_{i} \in[\ell]^{i}$, we denote by $H^{(j)}\left[\Lambda_{i}\right]=H^{(j)}\left[\bigcup_{\lambda \in \Lambda_{i}} V_{\lambda}\right]$ the subhypergraph of the $(m, \ell, j)$-hypergraph $H^{(j)}$ induced on $\bigcup_{\lambda \in \Lambda_{i}} V_{\lambda}$.

For an $(m, \ell, j)$-hypergraph $H^{(j)}$ and an integer $i, j \leq i \leq \ell$, we denote by $\mathcal{K}_{i}\left(H^{(j)}\right)$ the set of all $i$-subsets of $V\left(H^{(j)}\right)$ which span complete subhypergraphs in $H^{(j)}$ on $i$ vertices. Note that $\left|\mathcal{K}_{i}\left(H^{(j)}\right)\right|$ is the number of all copies of $K_{i}^{(j)}$ in $H^{(j)}$.

Given an $(m, \ell, j-1)$-hypergraph $H^{(j-1)}$ and an $(m, \ell, j)$-hypergraph $H^{(j)}$ such that $V\left(H^{(j)}\right) \subseteq V\left(H^{(j-1)}\right)$, we say that a hyperedge $J$ of $H^{(j)}$ belongs to $H^{(j-1)}$ if $J \in \mathcal{K}_{j}\left(H^{(j-1)}\right)$, i.e., $J$ corresponds to a clique of order $j$ in $H^{(j-1)}$. Moreover, $H^{(j-1)}$ underlies $H^{(j)}$ if $H^{(j)} \subseteq \mathcal{K}_{j}\left(H^{(j-1)}\right)$, i.e., every hyperedge of $H^{(j)}$ belongs to $H^{(j-1)}$. This brings us to the notion of a complex. Let $m \geq 1$ and $\ell \geq h \geq 1$ be integers. An $(m, \ell, h)$-complex $\boldsymbol{H}$ is a collection of $(m, \ell, j)$-hypergraphs $\left\{H^{(j)}\right\}_{j=1}^{h}$ such that

(a) $H^{(1)}$ is an $(m, \ell, 1)$-hypergraph, i.e., $V\left(H^{(1)}\right)=V_{1} \cup \cdots \cup V_{\ell}$ with $\left|V_{i}\right|=m$ for $i \in[\ell]$, and

(b) $H^{(j-1)}$ underlies $H^{(j)}$ for $2 \leq j \leq h$, i.e., $H^{(j)} \subseteq \mathcal{K}_{j}\left(H^{(j-1)}\right)$. 
Now we proceed with the notion of relative density of a $j$-uniform hypergraph with respect to a $(j-1)$-uniform hypergraph on the same vertex set. For a given $j$-uniform hypergraph $H^{(j)}$ and a $(j-1)$-uniform hypergraph $H^{(j-1)}$ on the same vertex set, we define the density of $H^{(j)}$ with respect to $H^{(j-1)}$ as

$$
d\left(H^{(j)} \mid H^{(j-1)}\right)=\left\{\begin{array}{cl}
\frac{\left|H^{(j)} \cap \mathcal{K}_{j}\left(H^{(j-1)}\right)\right|}{\left|\mathcal{K}_{j}\left(H^{(j-1)}\right)\right|} & \text { if }\left|\mathcal{K}_{j}\left(H^{(j-1)}\right)\right|>0 \\
0 & \text { otherwise }
\end{array}\right.
$$

We also use a notion of regularity of an $(m, j, j)$-hypergraph with respect to an $(m, j, j-1)$-hypergraph. Let reals $\varepsilon>0$ and $d_{j} \geq 0$ be given along with an $(m, j, j)$-hypergraph $H^{(j)}$ and an underlying $(m, j, j-1)$-hypergraph $H^{(j-1)}$. We say $H^{(j)}$ is $\left(\varepsilon, d_{j}\right)$-regular with respect to $H^{(j-1)}$ if whenever $Q^{(j-1)} \subseteq H^{(j-1)}$ satisfies

$$
\left|\mathcal{K}_{j}\left(Q^{(j-1)}\right)\right| \geq \varepsilon\left|\mathcal{K}_{j}\left(H^{(j-1)}\right)\right|, \quad \text { then } d\left(H^{(j)} \mid Q^{(j-1)}\right)=d_{j} \pm \varepsilon .
$$

More generally, we extend the notion of $\left(\varepsilon, d_{j}\right)$-regularity from $(m, j, j)$ hypergraphs to $(m, \ell, j)$-hypergraphs $H^{(j)}$. We say that an $(m, \ell, j)$-hypergraph $H^{(j)}$ is $\left(\varepsilon, d_{j}\right)$-regular with respect to an $(m, \ell, j-1)$-hypergraph $H^{(j-1)}$ if the restriction $H^{(j)}\left[\Lambda_{j}\right]=H^{(j)}\left[\bigcup_{\lambda \in \Lambda_{j}} V_{\lambda}\right]$ is $\left(\varepsilon, d_{j}\right)$-regular with respect to to the restriction $H^{(j-1)}\left[\Lambda_{j}\right]=H^{(j-1)}\left[\bigcup_{\lambda \in \Lambda_{j}} V_{\lambda}\right]$ for every $j$ subset $\Lambda_{j} \in[\ell]^{j}$.

We sometimes write $\varepsilon$-regular to mean $\left(\varepsilon, d\left(H^{(j)} \mid H^{(j-1)}\right)\right)$-regular and we also omit the $m$ in $(m, \ell, h)$-complex and $(m, \ell, h)$-hypergraph.

Finally, we close this subsection with the notion of a regular complex.

Definition $4\left((\varepsilon, \boldsymbol{d})\right.$-regular complex). Let $\varepsilon>0$ and let $\boldsymbol{d}=\left(d_{2}, \ldots, d_{h}\right)$ be a vector of non-negative reals. An $(m, \ell, h)$-complex $\boldsymbol{H}=\left\{H^{(j)}\right\}_{j=1}^{h}$ is $(\varepsilon, \boldsymbol{d})$-regular if $H^{(j)}$ is $\left(\varepsilon, d_{j}\right)$-regular with respect to $H^{(j-1)}$ for $j=2, \ldots, h$.

\subsection{Partitions}

The regularity lemmas $[29,28]$ provide a well-structured family of partitions $\mathscr{P}=\left\{\mathscr{P}^{(1)}, \ldots, \mathscr{P}^{(k-1)}\right\}$, where $\mathscr{P}^{(i)}$ is a partition of the set of all $i$-subsets of some vertex set. First we define the refinement of a partition. Suppose $A \supseteq B$ are sets, $\mathscr{A}$ is a partition of $A$, and $\mathscr{B}$ is a partition of $B$. We say $\mathscr{A}$ refines $\mathscr{B}$ and write $\mathscr{A} \prec \mathscr{B}$ if for every $\mathcal{A} \in \mathscr{A}$ there either exists a $\mathcal{B} \in \mathscr{B}$ such that $\mathcal{A} \subseteq \mathcal{B}$ or $\mathcal{A} \subseteq A \backslash B$. 
Let $k$ be a fixed integer and $V$ be a set of vertices. Throughout this paper we require a family of partitions $\mathscr{P}=\left\{\mathscr{P}^{(1)}, \ldots, \mathscr{P}^{(k-1)}\right\}$ on $V$ to satisfy properties which we are going to describe below (see Definition 5).

Let $\mathscr{P}^{(1)}=\left\{V_{1}, \ldots, V_{\mid \mathscr{P}(1)}\right\}$ be a partition of some vertex set $V$. For every $j, 1 \leq j \leq k$, let $\operatorname{Cross}_{j}\left(\mathscr{P}^{(1)}\right)$ be the family of all crossing $j$-subsets $J$ of $V$, i.e., the set of all $j$-subsets which satisfy $\left|J \cap V_{i}\right| \leq 1$ for every $V_{i} \in \mathscr{P}^{(1)}$.

Suppose that partitions $\mathscr{P}^{(i)}$ of $\operatorname{Cross}_{i}\left(\mathscr{P}^{(1)}\right)$ into sets of $(i, i)$-hypergraphs, i.e., $i$-partite $i$-uniform hypergraphs, have been defined for $i=$ $1, \ldots, j-1$. Then for every $(j-1)$-subset $I$ in $\operatorname{Cross}_{j-1}\left(\mathscr{P}^{(1)}\right)$ there exists a unique $\mathcal{P}^{(j-1)}=\mathcal{P}^{(j-1)}(I) \in \mathscr{P}(j-1)$ so that $I \in \mathcal{P}^{(j-1)}$. Moreover, for every $j$-subset $J$ in $\operatorname{Cross}_{j}\left(\mathscr{P}^{(1)}\right)$ we define the polyad of $J$

$$
\hat{\mathcal{P}}^{(j-1)}(J)=\bigcup\left\{\mathcal{P}^{(j-1)}(I): I \in[J]^{j-1}\right\} .
$$

In other words, $\hat{\mathcal{P}}^{(j-1)}(J)$ is the unique collection of $j$ partition classes of $\mathscr{P}^{(j-1)}$ in which $J$ spans a copy of $K_{j}^{(j-1)}$. Observe that $\hat{\mathcal{P}}^{(j-1)}(J)$ can be viewed as a $(j, j-1)$-hypergraph, i.e., a $j$-partite, $(j-1)$-uniform hypergraph. More generally, for $1 \leq i<j$, we set

$$
\hat{\mathcal{P}}^{(i)}(J)=\bigcup\left\{\mathcal{P}^{(i)}(I): I \in[J]^{i}\right\} \quad \text { and } \quad \mathcal{P}(J)=\left\{\hat{\mathcal{P}}^{(i)}(J)\right\}_{i=1}^{j-1} .
$$

We also refer to $\mathcal{P}(J)$ as the polyad of $J$ and it will always be clear from the context which definition is meant.

Next, we define $\hat{\mathscr{P}}^{(j-1)}$, the family of all polyads by

$$
\hat{\mathscr{P}}^{(j-1)}=\left\{\hat{\mathcal{P}}^{(j-1)}(J): J \in \operatorname{Cross}_{j}\left(\mathscr{P}^{(1)}\right)\right\} .
$$

Note that two polyads $\hat{\mathcal{P}}^{(j-1)}(J)$ and $\hat{\mathcal{P}}^{(j-1)}\left(J^{\prime}\right)$ are not necessarily distinct for different $j$-subsets $J$ and $J^{\prime}$. We view $\hat{\mathscr{P}}^{(j-1)}$ as a set and, consequently,

$$
\left\{\mathcal{K}_{j}\left(\hat{\mathcal{P}}^{(j-1)}\right): \hat{\mathcal{P}}^{(j-1)} \in \hat{\mathscr{P}}^{(j-1)}\right\}
$$

is a partition of $\operatorname{Cross}_{j}\left(\mathscr{P}^{(1)}\right)$. The structural requirement on the partition $\mathscr{P}^{(j)}$ of $\operatorname{Cross}_{j}\left(\mathscr{P}^{(1)}\right)$ is

$$
\mathscr{P}^{(j)} \prec\left\{\mathcal{K}_{j}\left(\hat{\mathcal{P}}^{(j-1)}\right): \hat{\mathcal{P}}^{(j-1)} \in \hat{\mathscr{P}}^{(j-1)}\right\} .
$$

In other words, we require that the set of cliques spanned by a polyad in $\hat{\mathscr{P}}^{(j-1)}$ is sub-partitioned in $\mathscr{P}^{(j)}$ and every partition class in $\mathscr{P}^{(j)}$ belongs 
to precisely one polyad in $\hat{\mathscr{P}}^{(j-1)}$. Note, that due to (4) we inductively infer that $\mathcal{P}(J)$ defined in $(3)$ is a $(j, j-1)$-complex.

Throughout this paper we also want to have control over the number of partition classes in $\mathscr{P}^{(j)}$, and more specifically, over the number of classes contained in $\mathcal{K}_{j}\left(\hat{\mathcal{P}}^{(j-1)}\right)$ for a fixed polyad $\hat{\mathcal{P}}^{(j-1)} \in \hat{\mathscr{P}}^{(j-1)}$. We render this precisely in the following definition.

Definition 5 (Family of partitions). Suppose $V$ is a set of vertices, $k \geq 2$ is an integer and $\boldsymbol{a}=\left(a_{1}, \ldots, a_{k-1}\right)$ is a vector of positive integers. We say $\mathscr{P}=\mathscr{P}(k-1, \boldsymbol{a})=\left\{\mathscr{P}^{(1)}, \ldots, \mathscr{P}^{(k-1)}\right\}$ is a family of partitions on $V$, if it satisfies the following:

(i) $\mathscr{P}^{(1)}$ is a partition of $V$ into $a_{1}$ classes, and

(ii) for $j=2, \ldots, k-1, \mathscr{P}^{(j)}$ is a partition of $\operatorname{Cross}_{j}\left(\mathscr{P}^{(1)}\right)$ satisfying:

$$
\begin{gathered}
\mathscr{P}^{(j)} \quad \text { refines }\left\{\mathcal{K}_{j}\left(\hat{\mathcal{P}}^{(j-1)}\right): \hat{\mathcal{P}}^{(j-1)} \in \hat{\mathscr{P}}^{(j-1)}\right\} \text { and } \\
\left|\left\{\mathcal{P}^{(j)} \in \mathscr{P}^{(j)}: \mathcal{P}^{(j)} \subseteq \mathcal{K}_{j}\left(\hat{\mathcal{P}}^{(j-1)}\right)\right\}\right|=a_{j} \text { for every } \hat{\mathcal{P}}^{(j-1)} \in \hat{\mathscr{P}}^{(j-1)} .
\end{gathered}
$$

Moreover, we say $\mathscr{P}=\mathscr{P}(k-1, \boldsymbol{a})$ is $t$-bounded, if $\max \left\{a_{1}, \ldots, a_{k-1}\right\} \leq t$.

\subsection{Equitability and regular hypergraphs}

In this subsection we introduce the notion of equitability.

Definition $6((\eta, \varepsilon, \boldsymbol{a})$-equitable). Suppose $V$ is a set of $n$ vertices, $\eta$ and $\varepsilon$ are positive reals, $\boldsymbol{a}=\left(a_{1}, \ldots, a_{k-1}\right)$ is a vector of positive integers, and $a_{1}$ divides $n$.

We say a family of partitions $\mathscr{P}=\mathscr{P}(k-1, \boldsymbol{a})=\left\{\mathscr{P}^{(1)}, \ldots, \mathscr{P}^{(k-1)}\right\}$ on $V$ is $(\eta, \varepsilon, \boldsymbol{a})$-equitable if it satisfies the following:

(a) $\left|[V]^{k} \backslash \operatorname{Cross}_{k}\left(\mathscr{P}^{(1)}\right)\right| \leq \eta\left(\begin{array}{l}n \\ k\end{array}\right)$, and

(b) $\mathscr{P}^{(1)}=\left\{V_{i}: i \in\left[a_{1}\right]\right\}$ is an equitable vertex partition, i.e., $\left|V_{i}\right|=$ $|V| / a_{1}$ for each $i \in\left[a_{1}\right]$, and

(c) for every $k$-subset $K \in \operatorname{Cross}_{k}\left(\mathscr{P}^{(1)}\right)$ we have $\mathcal{P}(K)=\left\{\hat{\mathcal{P}}^{(j)}(K)\right\}_{j=1}^{k-1}$ is an $(\varepsilon, \boldsymbol{d})$-regular $\left(n / a_{1}, k, k-1\right)$-complex with $\boldsymbol{d}=\left(1 / a_{2}, \ldots, 1 / a_{k-1}\right)$.

Note that from equitability one obtains an implicit bound $a_{1}>1 /(2 \eta)$ for $n$ sufficiently large.

Now we extend the definition of $\left(\varepsilon, d_{j}\right)$-regularity.

Definition $7\left(\left(\delta_{k}, d_{k}, f\right)\right.$-regular hypergraph). Let $\delta_{k}$ and $d_{k}$ be positive reals and $f$ be a positive integer. Suppose $H^{(k-1)}$ is an $(m, k, k-1)$-hypergraph spanning at least one $K_{k}^{(k-1)}$. We say that an $(m, k, k)$-hypergraph $H^{(k)}$ is 
$\left(\delta_{k}, d_{k}, f\right)$-regular with respect to $H^{(k-1)}$ if for every collection $\boldsymbol{Q}^{(k-1)}=$ $\left\{Q_{1}^{(k-1)}, \ldots, Q_{f}^{(k-1)}\right\}$ of not necessarily disjoint subhypergraphs of $H^{(k-1)}$ which satisfy

$$
\left|\bigcup_{i \in[f]} \mathcal{K}_{k}\left(Q_{i}^{(k-1)}\right)\right| \geq \delta_{k}\left|\mathcal{K}_{k}\left(H^{(k-1)}\right)\right|>0
$$

we have

$$
\frac{\left|H^{(k)} \cap \bigcup_{i \in[f]} \mathcal{K}_{k}\left(Q_{i}^{(k-1)}\right)\right|}{\left|\bigcup_{i \in[f]} \mathcal{K}_{k}\left(Q_{i}^{(k-1)}\right)\right|}=d_{k} \pm \delta_{k} .
$$

We write $\left(\delta_{k}, *, f\right)$-regular to mean $\left(\delta_{k}, d\left(H^{(k)} \mid H^{(k-1)}\right), f\right)$-regular. Moreover, if $f=1$, then a $\left(\delta_{k}, d_{k}, 1\right)$-regular hypergraph is $\left(\varepsilon, d_{k}\right)$-regular with $\varepsilon=\delta_{k}$ and vice versa.

Next we say when a hypergraph is regular with respect to a given family of partitions.

Definition $8\left(\left(\delta_{k}, *, f\right)\right.$-regular with respect to $\left.\mathscr{P}\right)$. Let $\delta_{k}$ be a positive real and $f$ a positive integer. Let $H^{(k)}$ be a $k$-uniform hypergraph with vertex set $V$ and $\mathscr{P}=\mathscr{P}(k-1, \boldsymbol{a})$ be a family of partitions on $V$. We say $H^{(k)}$ is $\left(\delta_{k}, *, f\right)$-regular with respect to $\mathscr{P}$, if

$$
\mid \bigcup\left\{\mathcal{K}_{k}\left(\hat{\mathcal{P}}^{(k-1)}\right): \hat{\mathcal{P}}^{(k-1)} \in \hat{\mathscr{P}}^{(k-1)}\right.
$$

and $H^{(k)}$ is not $\left(\delta_{k}, *, f\right)$-regular with respect to $\left.\hat{\mathcal{P}}^{(k-1)}\right\}\left.\left|\leq \delta_{k}\right| V\right|^{k}$.

If $H^{(k)}$ is $\left(\delta_{k}, *, f\right)$-regular with respect to $\hat{\mathcal{P}}^{(k-1)}$ then we call the polyad $\mathcal{P}(J)$ (also $\left.\hat{\mathcal{P}}^{(k-1)}\right)$ regular, where $J \in \mathcal{K}_{k}\left(\hat{\mathcal{P}}^{(k-1)}\right)$. Note, that $\mathcal{P}(J)=\mathcal{P}\left(J^{\prime}\right)$ for all $J$ and $J^{\prime}$ in $\mathcal{K}_{k}\left(\hat{\mathcal{P}}^{(k-1)}\right)$.

\subsection{Regularity and counting lemma}

Finally, we state the regularity lemma [29] we are going to use (see for example Lemma 23 in [29]).

Theorem 9 (Regularity lemma). Let $k \geq 2$ and $c \geq 1$ be fixed integers. For all positive constants $\eta$ and $\delta_{k}$, and all functions $f: \mathbb{N}^{k-1} \longrightarrow \mathbb{N}$ and $\delta: \mathbb{N}^{k-1} \longrightarrow(0,1]$ there are integers $t_{0}$ and $n_{0}$ so that the following holds. 
For every $k$-uniform hypergraph $H^{(k)}$, which is an edge-disjoint union* of c k-uniform hypergraphs $H^{(k)}=H_{1}^{(k)} \dot{\cup} \cdots \dot{\cup} H_{c}^{(k)}$ with $\left|V\left(H^{(k)}\right)\right|=n \geq n_{0}$ such that $\left(t_{0}\right)$ ! divides $n$, there exists a family of partitions $\mathscr{P}=\mathscr{P}\left(k-1, \boldsymbol{a}^{\mathscr{P}}\right)$ so that

(i) $\mathscr{P}$ is $\left(\eta, \delta\left(\boldsymbol{a}^{\mathscr{P}}\right), \boldsymbol{a}^{\mathscr{P}}\right)$-equitable and $t_{0}$-bounded and

(ii) $H_{i}^{(k)}$ is $\left(\delta_{k}, *, f\left(\boldsymbol{a}^{\mathscr{P}}\right)\right)$-regular with respect to $\mathscr{P}$ for every color $i \in[c]$.

We use the following lemma, its proof can be derived from Theorem 1.3 in [28], we also refer the interested reader to Chapter 9 of [30].

Theorem 10 (Counting lemma). For any integer $k \geq 2$, every $k$-uniform hypergraph $F$ and every positive constant $d_{k}>0$, there exists $\delta_{k}>0$ such that for every $d_{k-1}, \ldots, d_{2}>0$ with $1 / d_{i} \in \mathbb{N}$ for every $i=2, \ldots, k-1$ there are constants $\delta=\delta\left(d_{2}, \ldots, d_{k-1}\right)>0$ and positive integers $f=$ $f\left(d_{2}, \ldots, d_{k-1}\right)$ and $m_{0}$ such that the following holds.

Let $H^{(k)}$ be a $k$-uniform hypergraph on $n \geq a_{1} m_{0}$ vertices and let $\mathscr{P}(k-1$, $\left.\left(a_{1}, 1 / d_{2}, \ldots, 1 / d_{k-1}\right)\right)=\left\{\mathscr{P}^{(1)}, \ldots, \mathscr{P}^{(k-1)}\right\}$ be a family of partitions.

If for a copy $F^{\prime}$ of $F$ in the complete $k$-uniform hypergraph with vertex set as $V\left(H^{(k)}\right)$ the following conditions are satisfied

(i) For every $e \in E\left(F^{\prime}\right)$ the polyad $\mathcal{P}_{e}=\left\{\hat{\mathcal{P}}_{e}^{(i)}\right\}_{i=1}^{k-1}$ with $e \in \mathcal{K}_{k}\left(\hat{\mathcal{P}}_{e}^{(k-1)}\right)$ is a $\left(\delta,\left(d_{2}, \ldots, d_{k-1}\right)\right)$-regular $\left(n / a_{1}, k, k-1\right)$-complex, and

(ii) for every $e \in E\left(F^{\prime}\right)$ the hypergraph $H^{(k)}$ is $\left(\delta_{k}, d_{e}, f\right)$-regular with respect to $\hat{\mathcal{P}}_{e}^{(k-1)}$ for some $d_{e} \geq d_{k}$,

then the hypergraph $H^{(k)}$ contains at least one copy of $F$.

Roughly speaking, this theorem says that if a collection of sufficiently regular complexes of $H$ is in a natural correspondence to the hyperedges of some fixed hypergraph $F$, then the given hypergraph $H$ must contain a copy of $F$.

We will also use the following special case of the dense counting lemma (see from [16, Theorem 6.5]). Theorem 11 yields good estimates on the number of hyperedges contained in polyads of an equitable family of partitions (which will be given to us through an application of the regularity lemma, Theorem 9).

Theorem 11 (Dense counting lemma). Fix $k \geq 2$. For any $\gamma>0$ and any $d_{2}, \ldots, d_{k-1}>0$, there exist $\delta_{0}$ and $n_{0} \in \mathbb{N}$ for which the following

\footnotetext{
* equivalently, one can think of the set of hyperedges of $H^{(k)}$ being colored with $c$ colors
} 
assertion holds. If $0<\delta<\delta_{0}$ and $H$ is a $(\delta, \boldsymbol{d})$-regular $(k, k-1)$-complex on $V_{1} \dot{\cup} \cdots \dot{\cup} V_{k}$, where $\boldsymbol{d}=\left(d_{2}, \ldots, d_{k-1}\right)$ and $\left|V_{i}\right|=n \geq n_{0}$ for all $i$, then the number of $K_{k}^{(k-1)}$ s in $H$ is $(1 \pm \gamma) \cdot d_{k-1}^{\left(\begin{array}{c}k \\ k-1\end{array}\right)} \cdots d_{2}^{\left(\begin{array}{c}k \\ 2\end{array}\right)} n^{k}$.

\subsection{Cluster hypergraphs and slices}

Similarly as in the graph case, we study the "cluster hypergraph" of a given family of partitions. However, in the hypergraph setting the natural cluster hypergraph is a "multihypergraph" and for our purposes it suffices to analyze an appropriate subhypergraph without multiple hyperedges. Such a representative we call a slice.

In the following we describe a slice more formally. For a given family of partitions $\mathscr{P}=\mathscr{P}(k-1, \boldsymbol{a})$, for every pair $\left(V_{i}, V_{j}\right)$ of two vertex classes from $\mathscr{P}^{(1)}$ we choose precisely one element $\mathcal{P}$ from $\mathscr{P}^{(2)}$ such that $\mathcal{P} \subseteq \mathcal{K}_{2}\left(V_{i}, V_{j}\right)$. More generally, for every $(j, j-1)$ polyad $\hat{\mathcal{P}}^{(j-1)}$ (which is a $(j, j-1)$ hypergraph) formed by elements from the slice we select precisely one $(j, j)$ hypergraph $\mathcal{P}^{(j)}$ with $\mathcal{P}^{(j)} \subseteq \mathcal{K}_{j}\left(\hat{\mathcal{P}}^{(j-1)}\right)$ from $\mathscr{P}^{(j)}$ for the slice. Note that there are exactly

$$
\prod_{i=2}^{k-1} a_{i}^{\left(\begin{array}{c}
a_{1} \\
i
\end{array}\right)}
$$

slices in the family $\mathscr{P}(k-1, \boldsymbol{a})$.

Define for every slice $\mathcal{S}$ a $|\mathscr{P}(1)|$-partite $k$-uniform hypergraph $H(\mathcal{S})$ with the vertex partition $\mathscr{P}^{(1)}$ whose hyperedges are exactly those $k$-sets that correspond to regular polyads $\mathcal{P}(J)$, contained in the slice $\mathcal{S}$ and such that $H^{(k)} \cap \mathcal{K}_{k}\left(\hat{\mathcal{P}}^{(k-1)}(J)\right)$ has sufficiently large density (with respect to $\left.\mathcal{K}_{k}\left(\hat{\mathcal{P}}^{(k-1)}(J)\right)\right)$, say, at least $d_{k}$. Then a special case of Theorem 10 above states that if $H(\mathcal{S})$ contains some copy of $F$, then $H^{(k)}$ must contain a copy of $F$ too.

\section{Proof of Theorem 2}

We prove the result only for $r=3$, as the two-color-case is similar. (In fact, for $r=2$ the first part of Theorem 2 already follows from (1).) Also note, the first claim of the theorem stating

$$
c_{r, F}(n) \leq r^{\operatorname{ex}(n, F)+o\left(n^{k}\right)}
$$


does not need any assumption on the $s$-stability of $F$. However, $s$-stability is only used in the last paragraph of Case 1, see below. Therefore we prove both claims simultaneously.

Given $\varepsilon>0$, let $\omega>0$ be given that satisfies the $s$-stability condition in Definition 1 for $\varepsilon / 3$ and the hypergraph $F$. We choose positive $\xi$ and $\zeta$ such that

$$
4(\xi+\zeta) \leq \min \{\omega, \varepsilon / 3\} \quad \text { and } \quad h(k ! 4 \xi)+4 \xi \leq \frac{\pi_{F} \zeta}{k ! 2^{k-1} 88}
$$

where $h(y):=-y \log y-(1-y) \log (1-y)$ for $0<y<1$ is the entropy function. Now apply Theorem 10 with $F$ and $d_{k}=\xi$ obtaining $\delta_{k}>0$. We may assume that

$$
\delta_{k} \leq \xi / 3
$$

as setting $\delta_{k}$ smaller makes the complexes we consider more regular (and therefore the statements still hold). We choose $\eta>0$ as follows

$$
\eta \leq 2 \xi / 3
$$

so that for every $a \geq 1 /(2 \eta)$, if a hypergraph on $a$ vertices has at least

$$
\pi_{F}(1+\zeta / 88)\left(\begin{array}{l}
a \\
k
\end{array}\right)
$$

hyperedges, then it contains a copy of $F$. Note that because $\operatorname{ex}(n, F) /\left(\begin{array}{l}n \\ k\end{array}\right)$ is a monotone decreasing function, which converges to $\pi_{F}$, such choices are always possible. Recall also that $a_{1} \geq(1 / 2 \eta)$ for an equitable family of partitions (cf. property (a) of Definition 6 ). Next choose $\gamma>0$ so that

$$
\gamma \leq \min \{1 / 9, \zeta / 88\}
$$

Let $\delta: \mathbb{N}^{k-1} \rightarrow(0,1]$ and $f: \mathbb{N}^{k-1} \rightarrow \mathbb{N}$ be the functions guaranteed by Theorem 10. Without loss of generality, we may assume that the function $\delta$ is smaller then the function guaranteed by Theorem 11 and hence the number of cliques of size $k$ that are spanned by any $\left(\delta\left(\boldsymbol{a}^{\mathscr{P}}\right), \boldsymbol{d}\right)$-regular $\left(n / a_{1}, k, k-\right.$ 1)-complex lie in the range

$$
(1 \pm \gamma)\left(\frac{n}{a_{1}}\right)^{k} / \prod_{i=2}^{k-1} a_{i}^{\left(\begin{array}{l}
k \\
i
\end{array}\right)}
$$


The rôle of $\gamma$ will become clear later in (13). Roughly speaking, after we regularize the hypergraph under consideration, we need good estimates on the number of hyperedges a polyad can contain. For this we apply Theorem 11 to an equitable family of partitions, in particular the last "layer" of this family forms a very regular partition of the $(k-1)$-subsets with precision $\delta$.

Now, let $m_{0}$ be given by Theorem 10 and $t_{0}$ by Theorem 9 . Further we choose $n_{0}$ larger than $t_{0} \cdot m_{0}$ and another $n_{0}$ given again by Theorem 9 .

Consider a hypergraph $H$ on $n \geq n_{0}$ vertices with $c_{3, F}(H) \geq 3^{\operatorname{ex}(n, F)}$ (for the "furthermore"-part we consider $H \in\left\{H_{1}, \ldots, H_{s+1}\right\}$ ). We assume without loss of generality that $\left(t_{0}\right)$ ! divides $n$, as otherwise we may delete less than $\left(t_{0}\right)$ ! vertices and obtain a subhypergraph $H^{\prime} \subset H$ with

$$
c_{3, F}\left(H^{\prime}\right) \geq 3^{\operatorname{ex}(n, F)-O\left(n^{k-1}\right)},
$$

and it follows from the proof that the $O\left(n^{k-1}\right)$ term does not harm us at all.

So fix any 3-hyperedge-coloring $\varphi$ of $H$, with color classes $H_{\text {green }}, H_{\text {blue }}$, $H_{\text {red }}$, without a monochromatic subhypergraph $F$. Apply Theorem 9 with the parameters $k, c=3, \delta_{k}, \eta$ and the functions $f$ and $\delta$ specified above. We obtain from Theorem 9 an integer $t_{0}$ and a family of partitions $\mathscr{P}=$ $\mathscr{P}\left(k-1, \boldsymbol{a}^{\mathscr{P}}\right)$ such that the properties specified in Theorem 9 hold. Roughly speaking, we know that $H_{\text {green }}, H_{\text {blue }}$, and $H_{\text {red }}$ are $\left(\delta_{k}, *, f\right)$-regular with respect to the obtained family of partitions.

We discard from our consideration the following colored hyperedges in $H$.

- all hyperedges which are not in $\operatorname{Cross}_{k}\left(\mathscr{P}^{(1)}\right)$, which are at most $\eta\left(\begin{array}{l}n \\ k\end{array}\right)$, and

- all hyperedges which are contained in $\left(\delta_{k}, *, f\left(\boldsymbol{a}^{\mathscr{P}}\right)\right)$-irregular polyads with respect to one of the colors, hence at most

$$
3 \delta_{k}|V|^{k}=3 \delta_{k} n^{k}
$$

such hyperedges, and

- furthermore, for every color we discard all hyperedges that are contained in $\left(\delta_{k}, *, f\left(\boldsymbol{a}^{\mathscr{P}}\right)\right)$-regular polyads of density less than $\xi$, which are at most $3 \xi\left(\begin{array}{l}n \\ k\end{array}\right)$.

So, in total we discard at most

$$
\eta\left(\begin{array}{l}
n \\
k
\end{array}\right)+3 \delta_{k} n^{k}+3 \xi\left(\begin{array}{l}
n \\
k
\end{array}\right) \leq 4 \xi n^{k}
$$

hyperedges, where we used (7) and (8). 
There are

$$
N_{p}:=\left(\begin{array}{c}
a_{1} \\
k
\end{array}\right) \cdot \prod_{i=2}^{k-1} a_{i}^{\left(\begin{array}{c}
k \\
i
\end{array}\right)}
$$

$(k, k-1)$ polyads in the partition $\mathscr{P}\left(k-1, \boldsymbol{a}^{\mathscr{P}}\right)$. Due to the choice of $\delta$, in every polyad $\mathcal{P}(J)$ there are at most

$$
E_{p}^{+}:=(1+\gamma)\left(\frac{n}{a_{1}}\right)^{k} / \prod_{i=2}^{k-1} a_{i}^{\left(\begin{array}{c}
k \\
i
\end{array}\right)}
$$

many hyperedges in each of the three colors, red, blue and green, due to Theorem 11.

Let $p_{\text {green }}, p_{\text {blue }}, p_{\text {red }}$ denote the number of $\left(\delta_{k}, *, f\left(\boldsymbol{a}^{\mathscr{P}}\right)\right)$-regular polyads of density at least $\xi$ in the colors green, blue and red, respectively. We know that every "monochromatic" slice cannot have more than ex $\left(a_{1}, F\right)$ such regular polyads, as otherwise, the counting lemma, Theorem 10, would imply that the hypergraph $H$ contains a monochromatic copy of $F$ which contradicts our choice of the coloring of the set of hyperedges of $H$.

Note that there are exactly

$$
S:=\prod_{i=2}^{k-1} a_{i}^{\left(\begin{array}{c}
a_{1} \\
i
\end{array}\right)}
$$

different slices, while every polyad occurs in exactly

$$
S \cdot \prod_{i=2}^{k-1} a_{i}^{-\left(\begin{array}{c}
k \\
i
\end{array}\right)}
$$

many slices.

Thus, we infer by averaging for every color col $\in\{$ green, blue, red $\}$ that the number $p_{\text {col }}$ of polyads satisfies

$$
p_{\mathrm{col}} \leq \operatorname{ex}\left(a_{1}, F\right) \cdot \prod_{i=2}^{k-1} a_{i}^{\left(\begin{array}{c}
k \\
i
\end{array}\right)}
$$

On the other hand, let $e_{j}$ for $j \in[3]$ denote the number of $\left(\delta_{k}, *, f\left(\boldsymbol{a}^{\mathscr{P}}\right)\right)$ regular polyads of density at least $\xi$ in exactly $j$ colors. We note the following 
simple identity

$$
e_{1}+2 e_{2}+3 e_{3}=p_{\text {green }}+p_{\text {red }}+p_{\text {blue }} \stackrel{(14)}{\leq} 3 \operatorname{ex}\left(a_{1}, F\right) \cdot \prod_{i=2}^{k-1} a_{i}^{\left(\begin{array}{c}
k \\
i
\end{array}\right)} .
$$

Now we split our argument into two parts.

Case 1. Assume first that

$$
e_{3} \geq(1-\zeta) \operatorname{ex}\left(a_{1}, F\right) \cdot \prod_{i=2}^{k-1} a_{i}^{\left(\begin{array}{l}
k \\
i
\end{array}\right)}
$$

which means by (12) and (15) that the number of hyperedges contained in polyads which are regular in at most two colors is at most

$$
\begin{aligned}
3 \zeta \operatorname{ex}\left(a_{1}, F\right) \cdot \prod_{i=2}^{k-1} a_{i}^{\left(\begin{array}{c}
k \\
i
\end{array}\right)} \cdot E_{p}^{+} & \leq 3 \zeta(1+\gamma) \cdot \frac{\operatorname{ex}\left(a_{1}, F\right)}{a_{1}^{k}} \cdot n^{k} \\
& \stackrel{(10)}{\leq} 4 \zeta \cdot \frac{\operatorname{ex}\left(a_{1}, F\right)}{a_{1}^{k}} \cdot n^{k} \leq 4 \zeta n^{k}
\end{aligned}
$$

We also discard these hyperedges. Hence, in view of (11), we discard at most $4(\zeta+\xi) n^{k}$ hyperedges in this case.

For a moment we ignore the different colors. We denote by $H^{\prime}$ the resulting hypergraph (consisting of the leftover hyperedges). The number of hyperedges in $H^{\prime}$ is at least $\operatorname{ex}(n, F)-4(\zeta+\xi) n^{k} \geq \pi_{F}\left(\begin{array}{l}n \\ k\end{array}\right)-\omega n^{k}$ (this follows trivially as $e(H) \geq \operatorname{ex}(n, F)$, which is again implied by $\left.c_{3, F}(n) \geq 3^{\operatorname{ex}(n, F)}\right)$. On the other hand, $H^{\prime}$ itself cannot have more than $\operatorname{ex}(n, F)$ hyperedges. Otherwise, there would exist a copy $F^{\prime}$ of $F$ in $H^{\prime}$. This is however impossible as then Theorem 10 applies, which yields a copy of $F$ even in every color. Indeed, the hyperedges of $F^{\prime}$ must lie in regular polyads of density at least $\xi$. Thus, conditions $(i)-(i i)$ are fulfilled, and we therefore find a copy of $F$ in $H$ in any color, which is a contradiction. Thus, $H^{\prime}$ is $F$-free and $e\left(H^{\prime}\right) \leq \operatorname{ex}(n, F)$.

From here, the first claim of the theorem follows immediately in view of the fact $e(H) \leq e\left(H^{\prime}\right)+4(\zeta+\xi) n^{k}<\operatorname{ex}(n, F)+\varepsilon n^{k}$.

As for the second claim, we argue as follows. If (16) holds for every $H \in\left\{H_{1}, \ldots, H_{s+1}\right\}$, then for every $i \in[s+1]$ there exists $H_{i}^{\prime} \subset H_{i}, H_{i}^{\prime}$ is $F$-free and $e\left(H_{i}^{\prime}\right) \geq \pi_{F}\left(\begin{array}{l}n \\ k\end{array}\right)-\omega n^{k}$. We infer from the $s$-stability that there exist $i, j \in[s+1], i \neq j$ such that $H_{i}^{\prime}$ and $H_{j}^{\prime}$ are $\varepsilon / 3$-close. Also we surely 
have that $\left|H_{i} \Delta H_{i}^{\prime}\right| \leq \varepsilon n^{k} / 3$ and $\left|H_{j} \Delta H_{j}^{\prime}\right| \leq \varepsilon n^{k} / 3$, which implies that $H_{i}$ and $H_{j}$ are $\varepsilon$-close. This finishes the first case and we conclude the theorem in this case.

Case 2. Now, we argue that under the assumption $c_{3, F}(H) \geq 3^{\operatorname{ex}(n, F)}$ there always exists some coloring, for which inequality (16) holds. We then arrive at a contradiction assuming that this is not the case.

So assume that (16) does not hold for any 3-hyperedge coloring of $H$ without a monochromatic copy of $F$. We can regularize the hypergraph $H$ for every hyperedge coloring. Our goal is to show, that $H$ cannot have too many hyperedge colorings.

We first bound the number of different $\left(\eta, \delta\left(\boldsymbol{a}^{\mathscr{P}}\right), \boldsymbol{a}^{\mathscr{P}}\right)$-equitable families of partitions which are $t_{0}$-bounded together with $\left(\delta_{k}, *, f\left(\boldsymbol{a}^{\mathscr{P}}\right)\right)$-regular polyads in every color of density at least $\xi$, and due to the $t_{0}$-boundedness there are at most

$$
\left(\prod_{i=1}^{k-1} t_{0}^{\left(\begin{array}{l}
n \\
i
\end{array}\right)}\right) \cdot 2^{3 N_{p}} \leq t_{0}^{2 n^{k-1}}
$$

of these. We also discarded at most $4 \xi n^{k}$ (cf. (11)) many hyperedges from irregular and "sparse" polyads, over which we had no control, thus we upper bound the number of ways one can additionally choose and color these hyperedges by

$$
\left(\begin{array}{c}
\left(\begin{array}{c}
n \\
k
\end{array}\right) \\
4 \xi n^{k}
\end{array}\right) \cdot 3^{4 \xi n^{k}} \leq 2^{h(k ! 4 \xi) n^{k}} \cdot 3^{4 \xi n^{k}}
$$

Now we are left to estimate the number of ways we can color the set of remaining hyperedges for some fixed family of partitions $\mathscr{P}$. There are at most

$$
\left(1^{e_{1}} \cdot 2^{e_{2}} \cdot 3^{e_{3}}\right)^{E_{p}^{+}}
$$

many ways, where we consider all possible hyperedges a polyad can span and take into account in how many colors some particular polyad is regular.

Set $T_{a}:=\prod_{i=2}^{k-1} a_{i}^{\left(\begin{array}{c}k \\ i\end{array}\right)}$. By assumption, $e_{3}<(1-\zeta) \operatorname{ex}\left(a_{1}, F\right) T_{a}$ and hence by (15) we have

$$
e_{2} \leq \frac{3}{2}\left(\operatorname{ex}\left(a_{1}, F\right) T_{a}-e_{3}\right)
$$


With $2<3^{7 / 11}$ we can upper bound (19) by

$$
\begin{aligned}
\left(2^{\frac{3}{2}\left(\operatorname{ex}\left(a_{1}, F\right) T_{a}-e_{3}\right)} \cdot 3^{e_{3}}\right)^{E_{p}^{+}} & \leq\left(3^{(21 / 22) \operatorname{ex}\left(a_{1}, F\right) T_{a}+(1 / 22) e_{3}}\right)^{E_{p}^{+}} \\
& \leq\left(3^{(21 / 22) \operatorname{ex}\left(a_{1}, F\right) T_{a}+(1 / 22)(1-\zeta) \operatorname{ex}\left(a_{1}, F\right) T_{a}}\right)^{E_{p}^{+}} \\
& =\left(3^{\operatorname{ex}\left(a_{1}, F\right) T_{a}-(1 / 22) \zeta \operatorname{ex}\left(a_{1}, F\right) T_{a}}\right)^{E_{p}^{+}} \\
& \stackrel{(13)}{\leq} 3^{(1-\zeta / 22)(1+\gamma) \operatorname{ex}\left(a_{1}, F\right)\left(n / a_{1}\right)^{k}}
\end{aligned}
$$

So, together with (17), (18) and (19), we upper bound the number $c_{3, F}(H)$ by

$$
\begin{aligned}
& t_{0}^{2 n^{k-1}} \cdot 2^{h(k ! 4 \xi) n^{k}} \cdot 3^{4 \xi n^{k}} \cdot 3^{(1-\zeta / 22)(1+\gamma) \operatorname{ex}\left(a_{1}, F\right)\left(n / a_{1}\right)^{k}} \\
& \quad \stackrel{(9)}{\leq} t_{0}^{2 n^{k-1}} 3^{h(k ! 4 \xi) n^{k}+4 \xi n^{k}+(1-\zeta / 22)(1+\gamma)(1+\zeta / 88) \pi_{F}\left(\begin{array}{c}
a_{1} \\
k
\end{array}\right)\left(n / a_{1}\right)^{k}} \\
& \quad \stackrel{(6),(10)}{\leq} 3^{\operatorname{ex}(n, F)-\zeta \pi_{F} n^{k} /(k ! 88)},
\end{aligned}
$$

and this contradicts the assumptions of the theorem and finishes the proof, because we have shown that Case 2 never occurs, and therefore always Case 1 applies.

We note that, in fact, we proved here a slightly stronger result which reads as follows:

Theorem 12. Let $k, s \in \mathbb{N}, k \geq 2$ and $r=2$ or 3 . Let $F$ be a $k$-uniform hypergraph, such that $\pi_{F}>0$. Furthermore suppose that $F$ is s-stable. Then, for every $\varepsilon>0$ there exist $\alpha>0$ and $n_{0} \in \mathbb{N}$ such that the following holds.

Among any $s+1$ many $k$-uniform hypergraphs $H_{1}, \ldots, H_{s+1}$ on $n \geq n_{0}$ vertices that satisfy $c_{r, F}\left(H_{i}\right) \geq r^{\operatorname{ex}(n, F)-\alpha n^{k}}$ for every $i \in[s+1]$, there exist two which are $\varepsilon$-close.

\section{Useful notations for Theorem 3}

Let $H=(V, E)$ be a $k$-uniform hypergraph and let $V_{1}, \ldots, V_{k} \subseteq V$ be $k$ mutually disjoint subsets of $V$. We denote by $E_{H}\left(V_{1}, \ldots, V_{k}\right)$ the set of all hyperedges in $H$ that intersect every subset $V_{i}, i \in[k]$, in exactly one vertex, and its cardinality is denoted by $e\left(V_{1}, \ldots, V_{k}\right):=\left|E_{H}\left(V_{1}, \ldots, V_{k}\right)\right|$. For a $t$ element set $\left\{v_{1}, \ldots, v_{t}\right\}$ of pairwise distinct vertices let $L_{H}\left(v_{1}, \ldots, v_{t}\right)$ be the 
set of all $(k-t)$-element subsets $S \subseteq V$, such that $v_{1}, \ldots, v_{t}$ together with $S$ form a hyperedge in the $k$-uniform hypergraph $H$. We occasionally call $L_{H}\left(v_{1}, \ldots, v_{t}\right)$ the $(k-t)$-uniform common link hypergraph (or graph).

By $\delta(H)$ we denote the minimum vertex degree of $H$, that is the minimum possible size of the set of hyperedges in $L_{H}(v)$ taken over all vertices $v \in H$.

By $t_{\ell}^{(k)}(n)$ denote the number of hyperedges in the Turán hypergraph $\mathcal{T}_{\ell}^{(k)}(n)$, thus the obvious lower and upper bounds are:

$$
\left(\begin{array}{l}
\ell \\
k
\end{array}\right) \cdot\left\lfloor\frac{n}{\ell}\right\rfloor^{k} \leq t_{\ell}^{(k)}(n) \leq\left(\begin{array}{l}
\ell \\
k
\end{array}\right) \cdot\left\lceil\frac{n}{\ell}\right\rceil^{k}
$$

and, moreover, we have the following lower bound on the minimum degree $\delta\left(\mathcal{T}_{\ell}^{(k)}(n)\right)$ :

$$
\delta\left(\mathcal{T}_{\ell}^{(k)}(n)\right) \geq\left(\begin{array}{l}
\ell-1 \\
k-1
\end{array}\right) \cdot\left\lfloor\frac{n}{\ell}\right\rfloor^{k-1}
$$

For a given $k$-uniform hypergraph $H$, a partition $V=V_{1} \dot{\cup} \cdots \dot{\cup} V_{\ell}$ of its vertex set into $\ell$ mutually disjoint classes, and any vertex, we distinguish between three different types of hyperedges incident to this vertex. Namely, for a vertex $v \in V_{j}$ for some $j \in[\ell]$ we refer to those hyperedges $e \in E$ incident to $v$ and intersecting every class $V_{i}, i \in[\ell]$, in at most one vertex as crossing hyperedges. Furthermore, hyperedges incident to vertex $v$, that intersect class $V_{j}$ in exactly one further vertex different from $v$ and else intersecting any other class $V_{i}, i \in[\ell] \backslash\{j\}$, in at most one vertex are referred to as defective hyperedges. Finally, the remaining hyperedges incident to vertex $v$ are called bad hyperedges. More formally, crossing hyperedges incident to vertex $v$ form the following subset of the set $E$ of hyperedges:

$$
E_{\text {cross }}(v):=\left\{e \in E: v \in e \text { and } \forall i \in[\ell]:\left|e \cap V_{i}\right| \leq 1\right\} \text {, }
$$

while the set of defective hyperedges incident to vertex $v \in V_{j}$ is

$$
E_{\text {defect }}(v):=\left\{e \in E: v \in e \text { and }\left|e \cap V_{j}\right|=2 \text { and } \forall i \in[\ell] \backslash j:\left|e \cap V_{i}\right| \leq 1\right\} .
$$

The set of bad hyperedges incident to vertex $v \in V_{j}$ is defined as

$$
E_{\text {bad }}(v)=\{e \in E: \quad v \in e\} \backslash\left(E_{\text {cross }}(v) \dot{\cup} E_{\text {defect }}(v)\right),
$$


or, equivalently

$E_{\mathrm{bad}}(v):=\left\{e \in E: v \in e\right.$ and $\left|e \cap V_{j}\right| \geq 3$ or $\exists i \in[\ell] \backslash\{j\}$ with $\left.\left|e \cap V_{i}\right| \geq 2\right\}$.

Let $\mathcal{P}$ be a partition of the vertex set $V$ with $V=V_{1} \dot{\cup} \ldots \dot{\cup} V_{\ell}, V_{1}, \ldots V_{\ell} \neq$ $\varnothing$. Let $\tau:[\ell] \rightarrow\{0,1, \ldots, k\}$ be a function such that $\sum_{i=1}^{\ell} \tau(i)=k$. Then, for a $k$-element subset (hyperedge) $e$ of $V$ we say that $e$ is of type $\tau$, if $\left|e \cap V_{i}\right|=\tau(i)$ for all $i \in[\ell]$. We thus may specify different types of hyperedges via their types. Therefore, a crossing hyperedge has type $\tau$, where exactly $k$ elements of $[\ell]$ are mapped to 1 and the remaining $(\ell-k)$ elements are mapped to 0 . Note that there are $\left(\begin{array}{c}k+\ell-1 \\ \ell-1\end{array}\right)$ different types of hyperedges with respect to the partition $V=V_{1} \dot{\cup} \cdots \dot{\cup} V_{\ell}$.

For a vertex $v \in V$ and a type $\tau$ associated with $v$ we write $\operatorname{deg}^{\tau}(v):=$ $\left|E^{\tau}(v)\right|$, where $E^{\tau}(v)$ denotes the set of all those hyperedges in $H$ of type $\tau$ which are incident to $v$.

\section{Generalized triangles $T_{3}$ and $T_{4}$}

Recall that $T_{k}$ denotes the $k$-uniform generalized triangle. Here we prove Theorem 3, namely, for $k=3$ or $k=4$, and $r=2$ or $r=3$, and $n$ sufficiently large it is

$$
c_{r, T_{k}}(n)=r^{\operatorname{ex}\left(n, T_{k}\right)} .
$$

However, due to the similarity of the arguments, we only give a proof in the case of $r=3$ colors and the 4 -uniform generalized triangle $T_{4}$. Recall that for $T_{4}$ and $n$ sufficiently large, the extremal hypergraph on $n$ vertices is the balanced, complete, 4-partite, 4-uniform Turán hypergraph $\mathcal{T}_{4}^{(4)}(n)$.

Proof of Theorem 3. Here we only prove the case $r=3$ and $k=4$.

Let $n_{0}$ be given by Theorem 2 (applied with $\delta$ as $\varepsilon$ ), we will specify $\delta$ below in (27) and let $n_{r, k}=n_{3,4} \geq n_{0}$ be sufficiently large.

The proof is similar to that in [21] and proceeds by contradiction. Assume that we are given a hypergraph $H$ on $n>n_{3,4}$ vertices with $c_{3, T_{4}}(H) \geq$ $3^{\operatorname{ex}\left(n, T_{4}\right)+m}$ for some $m \geq 0$. We show the following claim.

Claim 13. If $c_{3, T_{4}}(H) \geq 3^{\operatorname{ex}\left(n, T_{4}\right)+m}$ for some $m \geq 0$ and $H$ is not the Turán hypergraph $\mathcal{T}_{4}^{(4)}(n)$, then there exists an induced subhypergraph $H^{\prime}$ on $n^{\prime}$ vertices with $n^{\prime} \geq n-2$ and

$$
c_{3, T_{4}}\left(H^{\prime}\right) \geq 3^{\operatorname{ex}\left(n^{\prime}, T_{4}\right)+m+1} .
$$


Using Claim 13 (notice that $H^{\prime} \neq \mathcal{T}_{4}^{(4)}\left(n^{\prime}\right)$ ), inductively, we arrive at some subhypergraph $H_{0}$ of $H$ on at most $n_{0}$ vertices which admits at least $3^{\operatorname{ex}\left(n_{0}, T_{4}\right)+\left(\begin{array}{c}n_{0} \\ 4\end{array}\right)+1}$ monochromatic $T_{4}$-free 3 -colorings of the set of hyperedges, which is impossible and yields the desired contradiction. This proves Theorem 3, and thus, it is left to verify Claim 13.

Proof of Claim 13. Let $H$ be a hypergraph on $n$ vertices, $H \neq \mathcal{T}_{4}^{(4)}(n)$ and let $c_{3, T_{4}}(H) \geq 3^{\operatorname{ex}\left(n, T_{4}\right)+m}$ with $m \geq 0$. Clearly, this implies $e(H) \geq \operatorname{ex}\left(n, T_{4}\right)$.

Without loss of generality we may assume that the minimum degree of $H$ satisfies

$$
\delta(H) \geq \delta\left(\mathcal{T}_{4}^{(4)}(n)\right) \geq\left\lfloor\frac{n}{4}\right\rfloor^{3} .
$$

Otherwise, let $v$ be a vertex of minimum degree in $H$ and consider the subhypergraph $H^{\prime}:=H-\{v\}$. Since $\operatorname{ex}\left(n-1, T_{4}\right)=\operatorname{ex}\left(n, T_{4}\right)-\delta\left(\mathcal{T}_{4}^{(4)}(n)\right) \leq$ $\operatorname{ex}\left(n, T_{4}\right)-(\delta(H)+1)$ we have

$$
c_{3, T_{4}}\left(H^{\prime}\right) \geq \frac{c_{3, F}(H)}{3^{\delta(H)}} \geq 3^{\operatorname{ex}\left(n, T_{4}\right)-\delta(H)+m} \geq 3^{\operatorname{ex}\left(n-1, T_{4}\right)+m+1},
$$

which yields already (13). Consequently, from now on we may assume that $\delta(H) \geq \delta\left(\mathcal{T}_{4}^{(4)}(n)\right) \geq\lfloor n / 4\rfloor^{3}$.

Consider a partition $\mathcal{P}$ with $V(H)=V_{1} \dot{\cup} V_{2} \dot{\cup} V_{3} \dot{\cup} V_{4}$, which, among all partitions of $V(H)$ into four nonempty classes, maximizes $e_{H}\left(V_{1}, V_{2}, V_{3}, V_{4}\right)$, and therefore minimizes $e(H)-e_{H}\left(V_{1}, V_{2}, V_{3}, V_{4}\right)$. Since the generalized triangle $T_{4}$ is 1-stable, as proved by Pikhurko [26], by Theorem 2 we know that for our choice of $\delta>0$ we have

$$
e(H)-e_{H}\left(V_{1}, V_{2}, V_{3}, V_{4}\right)<\delta n^{4},
$$

which with (20) gives the upper bound $e(H) \leq\lceil n / 4\rceil^{4}+\delta n^{4}$ on the number of hyperedges in $H$. For $0<\delta \leq 1 / 4^{5}$ with $e(H) \geq \operatorname{ex}\left(n, T_{4}\right) \geq\lfloor n / 4\rfloor^{4}$, hence $e_{H}\left(V_{1}, V_{2}, V_{3}, V_{4}\right) \geq\lfloor n / 4\rfloor^{4}-\delta n^{4}$, we obtain the following lower and upper bounds on the sizes of the classes $V_{i}$ for all $i \in[4]$ :

$$
n / 4-3 \delta^{1 / 4} n \leq\left|V_{i}\right| \leq n / 4+3 \delta^{1 / 4} n .
$$

To see this, let $\left|V_{i}\right|=n / 4+3 p n / 4$ for some $i \in[4]$ and $p \geq 0$. Then, as the product of three positive numbers with given sum is maximal if all are the 
same, we must have (neglecting the roundings)

$$
\begin{aligned}
& \left(\frac{n}{4}+\frac{3 p}{4} n\right) \cdot\left(\frac{n}{4}-\frac{p}{4} n\right)^{3} \geq\left(\frac{n}{4}\right)^{4}-\delta n^{4} \\
& \Longleftrightarrow(1+3 p) \cdot(1-p)^{3} \geq 1-4^{4} \delta \\
& \Longrightarrow 1-3 p^{4} \geq 1-4^{4} \delta \quad \text { for } p \leq 3 / 4 \\
& \Longrightarrow 4 \delta^{1 / 4} \geq p,
\end{aligned}
$$

hence $\left|V_{i}\right| \leq n / 4+3 \delta^{1 / 4} n$. Moreover, since $(1+3 p)(1-p)^{3}$ is decreasing for $p \geq 0$, it is not possible that $p \geq 3 / 4$, as

$$
(1+3 p) \cdot(1-p)^{3} \leq \frac{13}{4} \cdot\left(\frac{1}{4}\right)^{3}=\frac{13}{4^{4}}<1-4^{4} \delta
$$

for $0<\delta \leq 1 / 4^{5}$.

On the other hand, if $\left|V_{i}\right|=n / 4-3 p n / 4$ for some $i \in[4]$ and $p \geq 0$, then as above we must have

$$
\begin{aligned}
& \left(\frac{n}{4}-\frac{3 p}{4} n\right) \cdot\left(\frac{n}{4}+\frac{p}{4} n\right)^{3} \geq\left(\frac{n}{4}\right)^{4}-\delta n^{4} \\
& \quad \Longleftrightarrow(1-3 p) \cdot(1+p)^{3} \geq 1-4^{4} \delta \\
& \quad \Longrightarrow 1-3 p^{4} \geq 1-4^{4} \delta \\
& \Longrightarrow 4 \delta^{1 / 4} \geq p,
\end{aligned}
$$

hence $\left|V_{i}\right| \geq n / 4-3 \delta^{1 / 4} n$.

Now our argument splits into three cases depending on the link hypergraph of a vertex. First we assume that there exists a vertex $v$ incident to at least $\beta n^{3}$ bad hyperedges with respect to the partition $\mathcal{P}$ of the vertex set $V(H)$ (Case 1). If this is not the case, then we assume that there exists a vertex $v$, which is incident to at least $\beta n^{3}$ defective hyperedges with respect to the partition $\mathcal{P}$ (Case 2). Finally, if neither Case 1 nor Case 2 holds, we deal with Case 3 , where every vertex is adjacent to at most $2 \beta n^{3}$ many defective or bad hyperedges. Thus, by assumption (23) on the minimum degree, since we choose $0<\beta \ll 1$ we know that every vertex is adjacent mostly to crossing hyperedges with respect to the partition $\mathcal{P}$.

For that we set $\beta, \delta>0$ as follows:

$$
\beta \leq \frac{7}{11} \cdot\left(\frac{1}{32}\right)^{3} \quad \text { and } \quad h(\beta / 12) \leq \frac{1}{9 \cdot 32^{3}} \quad \text { and } \quad \delta \leq \min \left\{(2 \beta)^{4}, \frac{1}{4^{24}}\right\},
$$


where $h(y)=-y \ln y-(1-y) \ln (1-y)$ for $0<y<1$ is the entropy function. However, it is sufficient to keep in mind that

$$
0<\delta \ll \beta \ll 1
$$

Case 1 ( $H$ satisfies $\exists i \in[4]$ and $\exists v \in V_{i}:\left|E_{\text {bad }}(v)\right| \geq \beta n^{3}$ ). Assume without loss of generality that $i=1$. Let $v \in V_{1}$ be a vertex such that $\left|E_{\text {bad }}(v)\right| \geq$ $\beta n^{3}$. Note that there are 16 types of bad hyperedges incident to vertex $v$. Thus, for at least one bad type $\tau$ we know $\left|E^{\tau}(v)\right| \geq \beta n^{3} / 16$. Therefore, there exists another vertex $w \neq v$ such that the common link graph $L_{H}(v, w)$ contains at least $\beta n^{2} / 16$ edges, which are contained in some class $V_{j}$ for some $j \in[4]$, that is, together with any edge from the link graph $L_{H}(v, w)$, the vertices $v$ and $w$ form a hyperedge of type $\tau$. Then we may find greedily a matching $M \subseteq\left(\begin{array}{c}V_{j} \\ 2\end{array}\right)$ and $M \subseteq L_{H}(v, w)$ of size at least $\beta n / 9$. Note here, that an already constructed matching of size $x$ can be extended as long as $2 x\left(n / 4+3 \delta^{1 / 4} n\right)<\beta n^{2} / 16$, hence we obtain a matching of size at least $\beta n / 9$ for $0<\delta \leq(1 / 97)^{4}$.

Now consider an edge $\{x, y\}$ from the matching $M$. For each $i \in[4] \backslash$ $\{j\}$ we may take each time one vertex $v_{i}$ from every class $V_{i} \backslash\{v, w\}$. Let these vertices be $v_{1}, v_{2}, v_{3}$. Then, for each such choice of $v_{1}, v_{2}, v_{3}$ these form together with vertex $x$ or $y$ a 4-element set. Moreover, adding the existing hyperedge $\{x, y, v, w\} \in E$, we obtain a copy of $T_{4}$, which is a subhypergraph of $H$ unless $\left\{x, v_{1}, v_{2}, v_{3}\right\}$ or $\left\{y, v_{1}, v_{2}, v_{3}\right\}$ is missing, i.e., is not a hyperedge in $H$. For $\delta \leq(1 / 96)^{4}$ and $n$ sufficiently large, there are at least $\left(n / 4-3 \delta^{1 / 4} n-2\right)^{3} \geq n^{3} / 100$ possibilities to choose such a triple $\left(v_{1}, v_{2}, v_{3}\right)$. Moreover, we may do this for any of the at least $\beta n / 9$ edges in $M$, each time obtaining distinct pairs of 4 -tuples $\left\{x, v_{1}, v_{2}, v_{3}\right\}$ and $\left\{y, v_{1}, v_{2}, v_{3}\right\}$, as each time we take another matching edge $\{x, y\}$. Since at most $\delta n^{4}$ hyperedges $\left\{x, v_{1}, v_{2}, v_{3}\right\}$ or $\left\{y, v_{1}, v_{2}, v_{3}\right\}$ are missing in $H$, we find for $0<\delta \leq \beta / 9,000$, which holds by (27), at least

$$
\left(n^{3} / 100\right)(\beta n / 9)-\delta n^{4} \geq \beta n^{4} / 1,000
$$

copies of $T_{4}$, which are subhypergraphs in $H$.

Now, let $F_{1}$ and $F_{2}$ be such distinct subhypergraphs $T_{4}$. Since $M$ is a matching, by our considerations from above we know that $F_{1}$ and $F_{2}$ either are hyperedge-disjoint, or they share a single hyperedge that consists of the vertices $v, w$ and a certain edge $e$ from the matching $M$. This hyperedge corresponds to the "third" hyperedge in the definition of the generalized triangle $T_{4}$, i.e., this hyperedge contains the symmetric difference of the first 
two. However, the point is that once the color of the hyperedge $\{v, w\} \cup e$ is fixed, we can color the two remaining hyperedges in each subhypergraph $T_{4}$ found in the described way in at most 8 instead of 9 ways, to exclude a monochromatic $T_{4}$. Applying the same considerations to all matching edges $e \in M$ with the corresponding subhypergraphs $T_{4}$, we obtain the following possibilities for coloring the set of hyperedges of $H$ :

- for every matching edge $e \in M$ the hyperedge $e \cup\{v, w\}$ may be colored in at most 3 ways,

- by (29) there exist at least $\beta n^{4} / 1,000$ pairwise distinct subhypergraphs $T_{4}$, and hence at least $2 \beta n^{4} / 1,000=\beta n^{4} / 500$ distinct hyperedges of $H$, such that two hyperedges of a single subhypergraph $T_{4}$ may be colored in at most 8 instead of 9 ways,

- finally, the set of remaining hyperedges may be colored arbitrarily by at most 3 colors.

This way, for $0<\delta \leq \beta / 10^{4}$, which holds by (27), and $n$ sufficiently large, with (25) we bound the number of 3 -colorings of the set of hyperedges of $H$ from above by

$$
3^{\operatorname{ex}\left(n, T_{4}\right)+\delta n^{4}-\beta n^{4} / 500} \cdot 8^{\beta n^{4} / 1,000} \ll 3^{\operatorname{ex}\left(n, T_{4}\right)},
$$

which contradicts the assumption $c_{3, T_{4}}(H) \geq 3^{\operatorname{ex}\left(n, T_{4}\right)}$.

Therefore, we have shown that Case 1 never holds, which we assume in the following.

Case 2 ( $H$ satisfies $\exists i \in[4]$ and $\exists v \in V_{i}:\left|E_{\text {defect }}(v)\right| \geq \beta n^{3}$ ). As we are not in Case 1, we know that $\forall v \in V:\left|E_{\text {bad }}(v)\right|<\beta n^{3}$.

Case 2 asserts a vertex $v \in V$ such that $\left|E_{\text {defect }}(v)\right| \geq \beta n^{3}$ with respect to the partition $\mathcal{P}$ with $V(H)=V_{1} \dot{\cup} V_{2} \dot{\cup} V_{3} \dot{\cup} V_{4}$. There are exactly 3 types of defective hyperedges incident to vertex $v$. Therefore, there exists a defective type $\tau$ such that $\left|E^{\tau}(v)\right| \geq \beta n^{3} / 3$. Without loss of generality we ssume that $v \in V_{1}$ and $\tau=(2,1,1,0)$. Recall that this means, that any defective hyperedge of type $\tau$ incident to vertex $v$ intersects class $V_{1}$ in another vertex distinct from $v$, and intersects also classes $V_{2}$ and $V_{3}$, but does not intersect class $V_{4}$, as $\tau(4)=0$. By the minimality of $e(H)-e_{H}\left(V_{1}, V_{2}, V_{3}, V_{4}\right)$, we know that

$$
\left|E_{\text {cross }}(v)\right| \geq \beta n^{3} / 3,
$$

otherwise, moving vertex $v$ to class $V_{4}$ would increase the number of crossing hyperedges, yielding at least $\beta n^{3} / 3$ crossing hyperedges. 
We also note that out of the 20 possible types $\tau$ of hyperedges incident to vertex $v$, we are left to consider only four, namely, the 3 defective and one crossing type of hyperedges. The amount of the other 16 types is less than $\beta n^{3}$.

We distinguish between two subsets of the set $\mathcal{C}$ of "allowed" colorings of the set of hyperedges of $H$. Let $\mathcal{C}_{1}$ consist of those hyperedge-colorings such that there exist two distinct types $\tau_{1}$ and $\tau_{2}$, either defective or crossing, with the following property: there exist subsets $E_{i}(v) \subset E^{\tau_{i}}(v)$ with $\left|E_{i}(v)\right| \geq$ $\beta n^{3} / 12$ for $i=1,2$, and both, $E_{1}(v)$ and $E_{2}(v)$, are monochromatic in the same color. Moreover, let $\mathcal{C}_{2}:=\mathcal{C} \backslash \overline{\mathcal{C}_{1}}$ be the set of remaining colorings.

We first show that $\left|\mathcal{C}_{1}\right| \leq 3^{\operatorname{ex}\left(n, T_{4}\right)-1}$, and then we concentrate on $\mathcal{C}_{2}$.

Consider a coloring from $\mathcal{C}_{1}$. By assumption, we always have at least two distinct (defective or crossing) types $\tau_{1}$ and $\tau_{2}$ with $\left|E^{\tau_{i}}(v)\right| \geq \beta n^{3} / 3$ for $i=1,2$. Let us assume that $\tau_{1}=\tau$ is the defective type described in the beginning, and let $\tau_{2}$ be another type. Here we give the arguments only when $\tau_{2}$ is the crossing type to simplify the presentation. The other cases can be easily treated in a similar way, which will be sketched at the end of this case.

Let $E_{i}(v) \subset E^{\tau_{i}}(v)$ with $\left|E_{i}(v)\right| \geq \beta n^{3} / 12, i=1,2$, be such that all hyperedges in $E_{1}(v) \dot{\cup} E_{2}(v)$ are colored by the same color, say green. Each set $E_{i}(v), i=1,2$, can be chosen in at most $\sum_{i=\beta n^{3} / 12}^{n^{3}}\left(\begin{array}{c}n^{3} \\ i\end{array}\right) \leq 2^{n^{3}}$ ways. With $\left|E_{1}(v)\right| \geq \beta n^{3} / 12$, and by (26) for $0<\delta \leq(1 / 96)^{4}$ there exists a pair $(u, w) \in V_{2} \times V_{3}$ such that $v, u, w$ are contained in at least $\beta n$ green distinct hyperedges intersecting class $V_{1}$ in another vertex different from $v$. We set $X:=\left\{x:\{x, u, v, w\} \in E_{2}(v)\right\}$. Furthermore, we know that $\left|E_{2}(v)\right| \geq$ $\beta n^{3} / 12$, and hence $\left|E_{2}(v) \cap E\left(V_{1}, V_{2} \backslash\{u\}, V_{3} \backslash\{w\}, V_{4}\right)\right| \geq \beta n^{3} / 13$ for $n$ sufficiently large. Thus, there are at least $\beta n^{3} / 13$ green crossing hyperedges incident to vertex $v$ and not containing the vertices $u$ or $w$. Let $f$ be such a crossing hyperedge and fix one of the at least $\beta n$ vertices $x \in X$. Then the 4-element set $g:=f \backslash\{v\} \dot{\cup}\{x\}$ together with the hyperedges $f$ and $\{x, v, u, w\}$ forms a subhypergraph $T_{4}$ unless $g$ is missing as a hyperedge. For $0<\delta \leq \beta^{2} / 200$, there are at least

$$
\left(\beta n^{3} / 13\right) \beta n-\delta n^{4}=\beta^{2} n^{4} / 13-\delta n^{4} \geq \beta^{2} n^{4} / 14
$$

many possibilities to choose such a hyperedge $g \in E$. Moreover, $g$ cannot be colored green, thus we only have two remaining colors that can be used. This way, for $n$ sufficiently large, we estimate the cardinality of the set $\mathcal{C}_{1}$ 
of colorings for $0<\delta<\beta^{2} / 40$ as follows:

$$
\begin{aligned}
\left|\mathcal{C}_{1}\right| & \leq 3 \cdot\left(\begin{array}{l}
4 \\
2
\end{array}\right) \cdot 2^{2 n^{3}} \cdot 3^{\operatorname{ex}\left(n, T_{4}\right)+\delta n^{4}-\beta^{2} n^{4} / 14} \cdot 2^{\beta^{2} n^{4} / 14} \\
& \leq 18 \cdot 2^{2 n^{3}} \cdot 3^{\operatorname{ex}\left(n, T_{4}\right)+\delta n^{4}-\beta^{2} n^{4} / 14} \cdot 2^{\beta^{2} n^{4} / 14} \\
& \leq 3^{\operatorname{ex}\left(n, T_{4}\right)-1}
\end{aligned}
$$

taking into account $\left(\begin{array}{l}4 \\ 2\end{array}\right)$ possibilities to choose the types $\tau_{1}$ and $\tau_{2}$, and 3 possibilities to choose the color of the hyperedges in the sets $E_{1}(v)$ and $E_{2}(v)$, where we used (25).

We now consider the colorings in $\mathcal{C}_{2}$. The most important observation is that, whenever we consider two different (defective or crossing) types $\tau_{1}$ and $\tau_{2}$ of hyperedges incident to vertex $v$, less than $\beta n^{3} / 12$ of the hyperedges from $E^{\tau_{i}}(v)$ can be colored by the same color for each $i \in[2]$. On the other hand, there are at least two (defective or crossing) types $\tau_{1}$ and $\tau_{2}$ for which $\left|E^{\tau_{i}}(v)\right| \geq \beta n^{3} / 3$. Thus, for each of these types $\tau_{i}, i=1,2$, there is a color $c_{i}$, which occurs at least $\beta n^{3} / 12$ often, where $c_{1} \neq c_{2}$. But then for each other (defective or crossing) type $\tau_{3}$ or $\tau_{4}$ each color $c_{1}$ and $c_{2}$ must occur less than $\beta n^{3} / 12$ often. Moreover, the third color $c, c \neq c_{1}, c_{2}$, may occur at least $\beta n^{3} / 12$ in at most one of the types. If this happens for type $\tau_{1}$ or $\tau_{2}$, then taking into account the at most $\beta n^{3}$ bad hyperedges incident to vertex $v$, there are at most

$$
\left(\begin{array}{l}
3 \\
2
\end{array}\right) \cdot 4 \cdot 3 \cdot 3^{\beta n^{3}} \cdot 2^{\left(n / 4+3 \delta^{1 / 4} n\right)^{3}} \cdot\left(\begin{array}{c}
n^{3} \\
\beta n^{3} / 12
\end{array}\right)^{9}
$$

colorings of the set of hyperedges of all types incident to vertex $v$, where we used $\sum_{0 \leq i<\beta n^{3} / 12} \leq\left(\begin{array}{c}n^{3} \\ \beta n^{3} / 12\end{array}\right)$.

Moreover, if color $c$ occurs at least $\beta n^{3} / 12$ often in type either $\tau_{3}$ or $\tau_{4}$, then there are at most

$$
\left(\begin{array}{l}
4 \\
3
\end{array}\right) \cdot 3 ! \cdot 3^{\beta n^{3}} \cdot\left(\begin{array}{c}
n^{3} \\
\beta n^{3} / 12
\end{array}\right)^{9}
$$

such colorings of all types of hyperedges incident to vertex $v$. Note that we first "choose" three types where some colors are present at least $\beta n^{3} / 12$ times and then we assign three colors to these type. Similarly it was argued in (34). 
Thus, for $n$ sufficiently large by (34) and (35) and our choice of the parameters $\beta, \delta>0$ in (27), we can estimate by using $2^{11 / 7}<3$ the number of ways the set of hyperedges incident to vertex $v$ can be colored by at most

$$
\begin{aligned}
37 & \cdot 3^{\beta n^{3}} \cdot 2^{\left(n / 4+3 \delta^{1 / 4} n\right)^{3}} \cdot\left(\begin{array}{c}
n^{3} \\
\beta n^{3} / 12
\end{array}\right)^{9} \\
& \leq 37 \cdot 3^{\beta n^{3}} \cdot 2^{\left(n / 4+3 \delta^{1 / 4} n\right)^{3}} \cdot 2^{9 h(\beta / 12) n^{3}} \\
& \leq 37 \cdot 3^{\beta n^{3}} \cdot 2^{(9 / 32)^{3} n^{3}} \cdot 2^{9 h(\beta / 12) n^{3}} \\
& \leq 37 \cdot 3^{\beta n^{3}+7 / 11(9 / 32)^{3} n^{3}} \cdot 3^{(63 / 11) h(\beta / 12) n^{3}} \\
& \leq 37 \cdot 3^{(7 / 11)\left(\left(9^{3}+2\right) / 32^{3}\right) n^{3}} \\
& \leq 3^{\delta\left(\mathcal{T}_{4}^{(4)}(n)\right)-1}
\end{aligned}
$$

since $\frac{7}{11}\left(9^{3}+2\right)<8^{3}$. Here we used the inequality $\left(\begin{array}{c}x \\ \alpha x\end{array}\right) \leq 2^{h(\alpha) x}$, where $h(y)$ is the entropy function. A simple averaging argument shows, that by deleting the vertex $v$ and all hyperedges incident to $v$ we obtain the hypergraph $H^{\prime}=H-\{v\}$ with

$$
c_{3, T_{4}}\left(H^{\prime}\right) \geq \frac{3^{\operatorname{ex}\left(n, T_{4}\right)+m}}{3^{\delta\left(\mathcal{T}_{4}^{(4)}(n)\right)-1}}=3^{\operatorname{ex}\left(n-1, T_{4}\right)+m+1},
$$

which yields (22) and concludes Case 2 for $\tau_{1}$ and $\tau_{2}$ as defined above.

Now assume that both types $\tau_{1}$ and $\tau_{2}$ are defective. For convenience, let without loss of generality $\tau_{1}=(2,1,1,0)$ and $\tau_{2}=(2,0,1,1)$. Similarly, we define the sets $E_{i}(v) \subset E^{\tau_{i}}(v), i=1,2$, of hyperedges of the same color, but now we fix a pair $(u, w) \in V_{1} \times V_{3}$ such that $v, u, w$ are contained in at least $\beta n$ green distinct hyperedges intersecting class $V_{4}$. Again, let $x \in V_{4}$ be such a vertex that forms a green hyperedge $\{v, u, w, x\}$, then it is not hard to see that $f \in E_{1}(v)$ together with $f \backslash\{v\} \cup\{x\}$ and $\{v, u, w, x\}$ form a potential copy of $T_{4}$. The rest of the argument remains valid.

Case 3 ( $H$ satisfies $\forall i \in[4]$ and $\left.\forall v \in V_{i}:\left|E_{\text {bad }}(v) \dot{\cup} E_{\text {defect }}(v)\right| \leq 2 \beta n^{3}\right)$. Here we are left with the last case, when Cases 1 and 2 do not hold, hence most of the hyperedges incident to any vertex $v$ are crossing. By assumption, $H \neq \mathcal{T}_{4}^{(4)}(n)$ and $c_{3, T_{4}}(H) \geq 3^{\operatorname{ex}\left(n, T_{4}\right)}$, hence there exists at least one noncrossing hyperedge $e$ with respect to the minimal partition $\mathcal{P}$ with $V(H)=$ $V_{1} \dot{\cup} V_{2} \dot{\cup} V_{3} \dot{\cup} V_{4}$. Let $u, v$ be two vertices that belong to this hyperedge $e$ and are contained in the same class. Recalling the minimum degree condition (23) for $H$, we infer with (26) that 


$$
\left|L_{\text {cross }}(u) \cap L_{\text {cross }}(v)\right| \geq 2\lfloor n / 4\rfloor^{3}-4 \beta n^{3}-\left(n / 4+3 \delta^{1 / 4} n\right)^{3},
$$

where for a vertex $w \in V$ it is $L_{\text {cross }}(w)=\left\{e \backslash\{w\}: e \in E_{\text {cross }}(w)\right\}$.

Subtracting from the right-hand side of (37) the term $n^{2}$, which is an upper bound on the number of triples in $L_{\text {cross }}(u) \cap L_{\text {cross }}(v)$ that intersect the hyperedge $e$ in a vertex different from $u$ and $v$, this way, for $n$ sufficiently large, we have identified at least

$$
2\lfloor n / 4\rfloor^{3}-4 \beta n^{3}-\left(n / 4+3 \delta^{1 / 4} n\right)^{3}-n^{2}
$$

subhypergraphs $T_{4}$, each two distinct of these sharing only the hyperedge $e$. We have for $0<\delta \leq(2 \beta)^{4}$ and $\delta \leq(1 / 12)^{4}$ and $n$ sufficiently large:

$$
\begin{aligned}
2\lfloor n / 4\rfloor^{3}-4 \beta n^{3}-\left(n / 4+3 \delta^{1 / 4} n\right)^{3}-n^{2} \\
\quad \geq 2(n / 4)^{3}-4 \beta n^{3}-\left(n / 4+3 \delta^{1 / 4} n\right)^{3}-3 n^{2} \\
\quad \geq 2(n / 4)^{3}-5 \beta n^{3}-\left(n / 4+3 \delta^{1 / 4} n\right)^{3} \\
\quad=(n / 4)^{3}-5 \beta n^{3}-(9 / 16) \delta^{1 / 4} n^{3}-(27 / 4) \delta^{1 / 2} n^{3}-27 \delta^{3 / 4} n^{3} \\
\quad \geq(n / 4)^{3}-8 \beta n^{3} .
\end{aligned}
$$

Given the color of the hyperedge $e$, the two other hyperedges of a fixed copy of $T_{4}$ may be colored in at most 8 instead of 9 ways. Therefore, for $n$ sufficiently large, by our choice (27) of $\beta, \delta>0$, with (38) and, using $\delta \leq 1 / 4^{24}$ and $\beta \leq 1 /\left(12 \cdot 4^{6}\right)$, we may estimate the number of ways of coloring all hyperedges incident to vertex $u$ or $v$ from above by

$$
\begin{aligned}
3 & \cdot 3^{4 \beta n^{3}} \cdot 8^{(n / 4)^{3}-8 \beta n^{3}} \cdot 3^{2\left[\left(n / 4+3 \delta^{1 / 4} n\right)^{3}-(n / 4)^{3}+8 \beta n^{3}\right]} \\
& =3 \cdot 3^{20 \beta n^{3}} \cdot 8^{(n / 4)^{3}-8 \beta n^{3}} \cdot 3^{2\left[\left(n / 4+3 \delta^{1 / 4} n\right)^{3}-(n / 4)^{3}\right]} \\
& =3 \cdot 3^{20 \beta n^{3}} \cdot 8^{(n / 4)^{3}-8 \beta n^{3}} \cdot 3^{9 \delta^{1 / 4} n^{3} / 8+27 \delta^{1 / 2} n^{3} / 2+54 \delta^{3 / 4}} n^{3} \\
& \leq 3 \cdot 3^{12 \beta n^{3}+3 \delta^{1 / 4} n^{3}} \cdot 8^{(n / 4)^{3}} \\
& \leq 3^{\delta\left(\mathcal{T}_{4}^{(4)}(n)\right)+\delta\left(\mathcal{T}_{4}^{(4)}(n-1)\right)-1} .
\end{aligned}
$$

Again, if we delete the vertices $u$ and $v$, we obtain the hypergraph $H^{\prime}=$ $H-\{u, v\}$, and, using a simple averaging argument, with (39) we obtain

$$
c_{3, T_{4}}\left(H^{\prime}\right) \geq 3^{\operatorname{ex}\left(n-2, T_{4}\right)+m+1} .
$$

This finishes the proof of Claim 13 and hence of Theorem 3. 


\section{The case of $r \geq 4$ colors}

In this section we prove lower bounds $c_{r, T_{3}}(n) \gg r^{\operatorname{ex}\left(n, T_{3}\right)}$ and $c_{r, T_{4}}(n) \gg$ $r^{\operatorname{ex}\left(n, T_{4}\right)}$ for $r \geq 4$.

First we consider the case of the 3 -uniform generalized triangle $T_{3}$. To prove a lower bound on $c_{r, T_{3}}(n)$ we give a lower bound on $c_{r, K_{3}}(n)$, i.e., for the case of graphs, where we forbid a monochromatic triangle [1]. Namely, consider the following graph $G=(V, E)$ with $|V|=n$ vertices, where without loss of generality $n$ is divisible by 4 . Let $V=V_{1} \dot{\cup} V_{2} \dot{\cup} V_{3} \dot{\cup} V_{4}$ be a partition of the vertex set $V$ with $\left|V_{i}\right|=n / 4, i \in[4]$, into equally sized classes. The edge set $E$ of $G$ consists of all edges $e=\{v, w\}$ with $v \in V_{i}$ and $w \in V_{j}$, where $i \neq j$. Given the set $[r], r \geq 4$, of colors, we color the set of all edges between classes $V_{1}$ and $V_{2}$, or between $V_{3}$ and $V_{4}$ by the colors $1, \ldots, r-1$. For the set of all edges between the classes $V_{1}$ and $V_{4}$, or $V_{2}$ and $V_{3}$ we use the colors $1, \ldots, r-2, r$. Moreover, the set of all edges between the classes $V_{1}$ and $V_{3}$, or $V_{2}$ and $V_{4}$ are colored arbitrarily by the colors $r-1$ and $r$. Here every coloring gives rise to a monochromatic bipartite graph, so no monochromatic triangle is created by the colorings described above.

The number of these colorings of the edge set of the graph $G$ for $r \geq 4$ is precisely

$$
\begin{aligned}
c_{r, K_{3}}(n) & \geq c_{r, K_{3}}(G)=(r-1)^{4\left(\frac{n}{4}\right)^{2}} \cdot 2^{2\left(\frac{n}{4}\right)^{2}} \\
& =((r-1) \cdot \sqrt{2})^{\frac{n^{2}}{4}} \\
& \gg r^{\frac{n^{2}}{4}} \geq r^{\operatorname{ex}\left(n, K_{3}\right)} .
\end{aligned}
$$

The lower bound (40) may be improved by using another distribution of the set $[r]$ of colors, namely for $r$ divisible by 3 say, we color the set of all edges between the classes $V_{1}$ and $V_{2}$, or $V_{3}$ and $V_{4}$ by the colors $1, \ldots, 2 r / 3$. For the set of all edges between the classes $V_{1}$ and $V_{4}$, or $V_{2}$ and $V_{3}$ we use the colors $1, \ldots, r / 3,2 r / 3+1, \ldots, r$. Moreover, the set of all edges between the classes $V_{1}$ and $V_{3}$, or $V_{2}$ and $V_{4}$ are colored arbitrarily by the colors $r / 3+1, \ldots, r$, which gives

$$
c_{r, K_{3}}(n) \geq\left(\left(\frac{2 r}{3}\right)^{\frac{3}{2}}\right)^{\frac{n^{2}}{4}} \gg r^{\mathrm{ex}\left(n, K_{3}\right)}
$$

colorings.

Now we consider the 3 -uniform generalized triangle $T_{3}$ and the 3 -uniform, 2-partite hypergraph $H_{3}=(V, E)$ on $|V|=n$ vertices, which is defined as 
follows. Let $V=V_{0} \cup \dot{U} V^{\prime}$ be a partition with $\left|V_{0}\right|=n / 3$ and $\left|V^{\prime}\right|=2 n / 3$. All hyperedges $e \in E$ contain exactly one vertex from $V_{0}$ and two vertices from $V^{\prime}$. On the set $V^{\prime}$ we place the graph $G$ from above with $m=2 n / 3$ vertices. For any hyperedge $e=\left\{v_{0}, v, w\right\} \in E$ with $e \cap V_{0}=\left\{v_{0}\right\}$ its link $\{v, w\}$ has to be an edge in the graph $G$. The hyperedge $e=\left\{v_{0}, v, w\right\}$ may be colored by some color by which the edge $\{v, w\}$ may be colored.

Using (40), this yields exactly

$$
\begin{aligned}
c_{r, T_{3}}(n) & \geq c_{r, T_{3}}\left(H_{3}\right)=\left(((r-1) \cdot \sqrt{2})^{\frac{(2 n / 3)^{2}}{4}}\right)^{\frac{n}{3}}=((r-1) \cdot \sqrt{2})^{\frac{n^{3}}{27}} \\
& \gg r^{\frac{n^{3}}{27}} \geq r^{\operatorname{ex}\left(n, T_{3}\right)}
\end{aligned}
$$

colorings for $r \geq 4$ and $n$ sufficiently large. Of course, (42) may be improved by using (41).

It remains to show that the hypergraph $H_{3}$ does not contain a generalized triangle $T_{3}$. If $\{a, b, c\},\{b, c, d\}$ and $\{a, d, e\}$ is a subhypergraph $T_{3}$ in $H_{3}$, then one of the two vertices $b$ or $c$, and $e$ must be contained in class $V_{0}$, say $b, e \in V_{0}$. But then the union of the links of the vertices $b$ and $d$ forms a triangle in the graph $G$. However, due to the construction of the colorings, there is no monochromatic triangle $T_{2}$ in $G$, hence no monochromatic triangle $T_{3}$.

Next we consider the 4-uniform generalized triangle $T_{4}$ and the 4-uniform, 2-partite hypergraph $H_{4}=(V, E)$ on $|V|=n$ vertices, which is defined as follows. Let $V=V_{0} \dot{\cup} V^{\prime}$ be a partition with $\left|V_{0}\right|=n / 4$ and $\left|V^{\prime}\right|=3 n / 4$. All hyperedges $e \in E$ contain exactly one vertex from $V_{0}$ and three vertices from $V^{\prime}$. On the set $V^{\prime}$ we place the hypergraph $H_{3}$ from above with $m=$ $3 n / 4$ vertices. For any hyperedge $e=\left\{v_{0}, v, w, x\right\} \in E$ with $e \cap V_{0}=\left\{v_{0}\right\}$ its link $\{v, w, x\}$ has to be a hyperedge in the hypergraph $H_{3}$. The hyperedge $e=\left\{v_{0}, v, w, x\right\}$ may be colored by some color by which the hyperedge $\{v, w, x\}$ in $H_{3}$ may be colored.

With (42), this gives

$$
\begin{aligned}
c_{r, T_{4}}(n) & \geq c_{r, T_{4}}\left(H_{4}\right)=\left(((r-1) \cdot \sqrt{2})^{\frac{(3 n / 4)^{3}}{27}}\right)^{\frac{n}{4}}=((r-1) \cdot \sqrt{2})^{\frac{n^{4}}{256}} \\
& \gg r^{\frac{n^{4}}{256}} \geq r^{\operatorname{ex}\left(n, T_{4}\right)}
\end{aligned}
$$

colorings for $r \geq 4$ and $n$ sufficiently large.

It remains to show that the hypergraph $H_{4}$ does not contain a generalized triangle $T_{4}$. If $\{a, b, c, d\},\{e, b, c, d\}$ and $\{a, e, f, g\}$ is a subhypergraph $T_{4}$ in 
$H_{4}$, then one of the three vertices $b, c$ or $d$, and $f$ or $g$ must be contained in class $V_{0}$, say $b, f \in V_{0}$. But then the union of the links of $b$ and $f$ forms a generalized triangle in the hypergraph $H_{3}$. However, due to the construction of the colorings, there is no monochromatic generalized triangle $T_{3}$, hence no monochromatic generalized triangle $T_{4}$.

\section{Concluding remarks}

There is a recent result of Balogh and Mubayi [4], who prove that almost all $T_{3}$-free 3 -uniform hypergraphs are tripartite. The first step in their approach (and in many other approaches to derive "good" bounds on $|\operatorname{Forb}(n, F)|$, where $F$ is some uniform hypergraph) is to apply an appropriate form of regularity lemma to show that almost all $T_{3}$-free hypergraphs are nearly tripartite, i.e., $\varepsilon$-close to some tripartite 3 -uniform hypergraph. It is conceivable, that we can modify the proof of Theorem 2 to yield a similar general result for $F$-free hypergraphs which admit some appropriate $s$-stability definition. In particular, we are quite confident that we can adjust the proof of Theorem 2 to show that almost all $T_{4}$-free 4 -uniform hypergraphs are $\varepsilon$-close to being 4-partite. It is also conceivable, that then, using some "selfimprovement" type of the argument, see e.g. [2, 24, 4], one should be able to show that almost all $T_{4}$-free hypergraphs are in fact 4 -partite.

We also would like to remark that, given Theorem 2, one needs just to look out for those hypergraphs that have been proven to be, say 1-stable and that, say, have unique extremal hypergraphs. There, one might be able to even prove an exact result similar to that of Theorem 3. In a forthcoming paper of the first two authors [20], other classes of hypergraphs are studied, and some exact results similar to that of Theorem 3 are proven there.

\section{References}

[1] N. Alon, J. Balogh, P. Keevash, and B. Sudakov, The number of edge colorings with no monochromatic cliques, J. London Math. Soc. (2) 70 (2004), no. 2, 273-288. MR2078893

[2] J. Balogh, B. Bollobás, and M. Simonovits, The number of graphs without forbidden subgraphs, J. Combin. Theory Ser. B 91 (2004), no. 1, 1-24. MR2047528

[3] J. Balogh, B. Bollobás, and M. Simonovits, The typical structure of graphs without given excluded subgraphs, Random Structures Algorithms 34 (2009), no. 3, 305-318. MR2504400 
[4] J. Balogh and D. Mubayi, Almost all cancellative triple systems are tripartite, to appear, Combinatorica.

[5] F. R. K. Chung, Regularity lemmas for hypergraphs and quasirandomness, Random Structures Algorithms 2 (1991), no. 2, 241-252. MR1099803

[6] P. Erdős, Some new applications of probability methods to combinatorial analysis and graph theory, Proceedings of the Fifth Southeastern Conference on Combinatorics, Graph Theory and Computing (Florida Atlantic Univ., Boca Raton, Fla., 1974) (Winnipeg, Man.), Utilitas Math., 1974, pp. 39-51. Congressus Numerantium, No. X. MR0364020

[7] P. Erdös, P. Frankl, and V. Rödl, The asymptotic number of graphs not containing a fixed subgraph and a problem for hypergraphs having no exponent, Graphs Combin. 2 (1986), 113-121. MR0932119

[8] P. Erdős, D. J. Kleitman, and B. L. Rothschild, Asymptotic enumeration of $K_{n}$-free graphs, Colloquio Internazionale sulle Teorie Combinatorie (Rome, 1973), Tomo II, Accad. Naz. Lincei, Rome, 1976, pp. 19 27. Atti dei Convegni Lincei, No. 17. MR0463020

[9] P. Frankl and Z. Füredi, A new generalization of the Erdös-Ko-Rado theorem, Combinatorica 3 (1983), no. 3-4, 341-349. MR0729787

[10] P. Frankl and Z. Füredi, Extremal problems whose solutions are the blowups of the small Witt-designs, J. Combin. Theory Ser. A 52 (1989), no. 1, 129-147. MR1008165

[11] P. Frankl and V. Rödl, The uniformity lemma for hypergraphs, Graphs Combin. 8 (1992), no. 4, 309-312. MR1204114

[12] Z. Füredi and M. Simonovits, Triple systems not containing a Fano configuration, Combin. Probab. Comput. 14 (2005), no. 4, 467-484. MR2160414

[13] P. Keevash and D. Mubayi, Stability theorems for cancellative hypergraphs, J. Combin. Theory Ser. B 92 (2004), no. 1, 163-175. MR2078500

[14] P. Keevash and B. Sudakov, The Turán number of the Fano plane, Combinatorica 25 (2005), no. 5, 561-574. MR2176425

[15] Y. Kohayakawa, B. Nagle, V. Rödl, and M. Schacht, Weak hypergraph regularity and linear hypergraphs, J. Combin. Theory Ser. B 100 (2010), no. 2, 151-160. MR2595699 
[16] Y. Kohayakawa, V. Rödl, and J. Skokan, Quasi-randomness, hypergraphs, and conditions for regularity, J. Combin. Theory Ser. A 97 (2002), no. 2, 307-352. MR1883869

[17] Ph. G. Kolaitis, H. J. Prömel, and B. L. Rothschild, Asymptotic enumeration and a 0-1 law for m-clique free graphs, Bull. Amer. Math. Soc. (N.S.) 13 (1985), no. 2, 160-162. MR0799802

[18] Ph. G. Kolaitis, H. J. Prömel, and B. L. Rothschild, $K_{l+1}$-free graphs: asymptotic structure and a $0-1$ law, Trans. Amer. Math. Soc. 303 (1987), no. 2, 637-671. MR0902790

[19] J. Komlós and M. Simonovits, Szemerédi's regularity lemma and its applications in graph theory, Combinatorics, Paul Erdős is Eighty, Vol. 2 (Keszthely, 1993), Bolyai Soc. Math. Stud., vol. 2, János Bolyai Math. Soc., Budapest, 1996, pp. 295-352. MR1395865

[20] H. Lefmann and Y. Person, Exact results on the number of restricted edge colorings for some families of linear hypergraphs, submitted.

[21] H. Lefmann, Y. Person, V. Rödl, and M. Schacht, On colorings of hypergraphs without monochromatic Fano planes, Combin. Probab. Comput. 18 (2009), no. 5, 803-818. MR2534270

[22] B. Nagle and V. Rödl, The asymptotic number of triple systems not containing a fixed one, Discrete Math. 235 (2001), no. 1-3, 271-290, Combinatorics (Prague, 1998). MR1829856

[23] B. Nagle, V. Rödl, and M. Schacht, Extremal hypergraph problems and the regularity method, Topics in Discrete Mathematics, Algorithms Combin., vol. 26, Springer, Berlin, 2006, pp. 247-278. MR2249275

[24] Y. Person and M. Schacht, Almost all hypergraphs without Fano planes are bipartite, Proceedings of the Twentieth Annual ACM-SIAM Symposium on Discrete Algorithms (C. Mathieu, ed.), ACM, 2009, pp. 217226.

[25] O. Pikhurko, Exact computation of the hypergraph Turán function for expanded complete 2-graphs, accepted to J. Combin. Theory Ser. B, but publication suspended because of a disagreement over the copyright.

[26] O. Pikhurko, An exact Turán result for the generalized triangle, Combinatorica 28 (2008), no. 2, 187-208. MR2399018

[27] O. Pikhurko and Z. B. Yilma, The maximum number of $K_{3}$-free and $K_{4}$-free edge 4-colorings, submitted. 
[28] V. Rödl and M. Schacht, Regular partitions of hypergraphs: counting lemmas, Combin. Probab. Comput. 16 (2007), no. 6, 887-901. MR2351689

[29] V. Rödl and M. Schacht, Regular partitions of hypergraphs: regularity lemmas, Combin. Probab. Comput. 16 (2007), no. 6, 833-885. MR2351688

[30] M. Schacht, On the regularity method for hypergraphs, Ph.D. thesis, Emory University, Department of Mathematics and Computer Science, May 2004. MR2705704

[31] M. Simonovits, A method for solving extremal problems in graph theory, stability problems, Theory of Graphs (Proc. Colloq., Tihany, 1966), Academic Press, New York, 1968, pp. 279-319. MR0233735

[32] A. Steger, Die Kleitman-Rothschild Methode, Ph.D. thesis, Forschungsinstitut für Diskrete Mathematik, Rheinische FriedrichWilhelms-Universität Bonn, March 1990.

[33] R. Yuster, The number of edge colorings with no monochromatic triangle, J. Graph Theory 21 (1996), no. 4, 441-452. MR1377618

HANNO LEFMANN

FAKULTÄT FÜR INFORMATIK

TeChnische Universität Chemnitz

Strasse DER NATIONEN 62

D-09107 ChEMNitz

GeRmany

E-mail address: Lefmann@Informatik.TU-Chemnitz.de

YuRY Person

INSTITUT FÜR INFORMATIK

HUMBOLDT-UNIVERSITÄt ZU BERLIN

UNTER DEN LINDEN 6

D-10099 BERLIN

Germany

E-mail address: person@informatik.hu-berlin.de

Mathias Schacht

Department Mathematik

UNIVERSITÄT HAMBURG

Bundesstrasse 55

D-20146 HAMBURG

Germany

E-mail address: schacht@math.uni-hamburg.de

ReCEIVEd April 3, 2010 